\title{
Capturing the molecular mechanism of anesthetic action using simulation methods
}

\author{
Victoria Oakes ${ }^{1}$ and Carmen Domene ${ }^{1,2^{*}}$ \\ ${ }^{1}$ Department of Chemistry, University of Bath, Claverton Down, Bath, BA2 7AY, UK \\ ${ }^{2}$ Department of Chemistry, University of Oxford, Oxford, OX1 3TA, Oxford, UK
}

Corresponding author: C.Domene@bath.ac.uk

Tel: +44 - (0) 1225386172

\section{Abstract}

Significant computational efforts have been focused towards exposing the molecular mechanisms of anesthesia in recent years. In the last decade, this has been aided considerably by a momentous increase in the number of high-resolution structures of ion channels, which are putative targets for the anesthetic agents, as well as advancements in high-performance computing technologies. In this review, typical simulation methods to investigate the behaviour of model membranes and membrane-protein systems are briefly reviewed, and related computational studies are surveyed. Both lipid and protein-mediated mechanisms of anesthetic action are scrutinized, focusing on the behaviour of ion channels in the latter case.

\section{Table of Contents}

1. Introduction

2. Common Simulation Methods

3. Anesthetic Interactions with Membranes

4. Anesthetic Interactions with Ion Channels

4.1 Pentameric ligand-gated ion channels or "Cys-loop" receptors.

4.2 Voltage-gated ion channels

\subsubsection{Sodium Channels}

\subsubsection{Potassium Channels}

5. Outlook 


\section{Introduction}

Anesthesia refers to a medically induced lack of sensation, enabling surgical procedures to take place in the absence of pain. During general anesthesia, the patient encounters a state of paralysis in the whole body, accompanied by unconsciousness. During local anesthesia, specific parts of the body are insensitive to pain, whilst patients are responsive. The identification of anesthetic molecules has revolutionized the medical industry, yet an in-depth understanding of the molecular mechanism of anesthetic action has not yet been achieved. Initial examinations by Meyer and Overton led to the proposal of a lipid-mediated mechanism for anesthesia. ${ }^{1,2}$ The early experimentalists revealed a notable correlation between the anesthetic potency and the solubility of molecules in oil, a lipid-like phase which is assumed to act as a simplified model of the membrane interior, advocating that anesthetics likely act via the plasma membrane. Studies of a variety of anesthetic compounds in an assortment of solvents have since been performed, ${ }^{3,4}$ and the Meyer-Overton hypothesis was upheld for nearly a century, until a number of notable exceptions were revealed. For example, Koblin and colleagues identified a series of polyhalogenated and perfluorinated compounds which displayed no anesthetic effect despite fulfilling the Meyer-Overton rule. ${ }^{5,6}$ On the other hand, the potency of short chains 1-alkanols was markedly underestimated by the rule. ${ }^{7}$

Several alternatives have now been proposed to account for such exceptions. Pohorille et al. identified an improved correlation when interfacial solubilities were used instead of bulk lipid solubilities, suggesting anesthetic compounds act at the lipid-water interface. ${ }^{8}$ Furthermore, Cantor discussed various membrane properties that could potentially modify the activity of intrinsic membrane proteins, recognizing that the specific composition can influence the lateral pressure profile of the membrane. ${ }^{9,10}$ As the conformational equilibria of membrane proteins is mechanically coupled to the lateral pressure profile, incorporation of compounds into the bilayer may therefore shift the activation state of membrane proteins such as ligand gated-ion channels. ${ }^{9,11}$ The effect of stiffness and interfacial activity of anesthetic molecules on the pressure distributions was used in this context to predict the potency of alkanols previously classified as anomalous by the Meyer-Overton rule. ${ }^{12}$

Alternative to the lipid-mediated theory, it has been postulated that anesthetics can act by directly binding to protein targets. Among early work, it was demonstrated that inhalational anesthetics directly inhibited the function of enzyme firefly luciferase by competitive binding of the substrate. ${ }^{13-15}$ Furthermore, anesthetics exerted an effect on soluble cytoplasmic proteins, such as 
protein kinase $C .{ }^{16,17}$ Considerable efforts were then focused towards identifying specific protein targets. Nowadays, specific classes of membrane receptors and membrane channels are considered to be dominant targets for anesthetics. Targets include GABA $A_{A}$, glycine, nicotinic acetylcholine, glutamate, adenosine and serotonin receptors, as well as voltage-gated, ATPsensitive and background potassium channels. ${ }^{18}$ These proteins are generally considered to accommodate anesthetic molecules in pockets/cavities, which consequently modulate their structure and dynamics in a specific manner. ${ }^{19}$ Thereupon, a legion of works has been published with the aim of classifying interaction sites and determining the repercussions of binding on the structure and dynamics of the protein.

Detailed understanding of membrane-ligand and membrane-protein interactions at an atomic level, and comprehension of the structural consequences of such binding, will greatly benefit future anesthetic drug design. In this review, we provide an overview of the achievements of computational tools for chemical biology in deciphering the molecular mechanism of anesthetic action. Firstly, a brief overview of common simulation methods used for the study of biological systems is given, and then we explore how such methodologies have been used to investigate both lipid and protein mediated theories. In the latter case, we focus on types of membranechannel systems, namely pentameric ligand gated ion channels and voltage-gated ion channels, which are both putative targets for anesthetics and have been studied extensively by computational means. We primarily focus on simulations based on high-resolution structural information, although homology models are mentioned where appropriate. Finally, a critical outlook on the state of the field will be surmised.

\section{Common simulation methods}

The broad range of tools for computational chemistry that are currently available enable researchers to study a considerable variety of properties. On the molecular level, molecular dynamics (MD) simulations are a popular method to analyze the dynamic behaviour of chemical entities in atomic detail. A brief overview of MD simulations and related methods are provided in this section. Further information concerning the mathematical background can be found in various textbooks on the topic. ${ }^{20-22}$ To perform MD simulations, the underlying potential energy of the system must be calculated in the first instance, via quantum or molecular mechanical methods. In the latter case, atoms and bonds are considered as balls and springs, respectively, thus nuclear motion is evaluated using classical physics, and an analytical expression for the energy of a system, known as a forcefield, can be utilized. Inter- and intramolecular energetic terms, including bond 
stretching, angle bending, bond rotations and non-bonded terms, contribute to the forcefield. This method in its simple version neglects electronic properties such as polarizabilities but permits the evaluation of a significant number of properties. Classical MD simulations are particularly suited to the study of biological systems, such as membrane proteins, due to the large number of atoms that are involved and the resolution of the events under investigation.

In order to obtain information about the time-dependent behaviour, the motion of interacting particles is calculated by the integrating Newton's equations of motion. The potential energy of the system, evaluated using the chosen forcefield, and the forces, evaluated using the negative gradient of the potential with respect to displacement in a specified direction, can be used to calculate the acceleration, and hence forecast the time evolution of the system, in the form of a trajectory. When few atoms are involved, analytical solutions of the equations of motion can be accessed, generating a continuous trajectory over time. In larger systems, this is unworkable due to a many body problem in force evaluations, from the existence of a continuous potential. Instead, finite difference methods can be used where forces are evaluated at discrete intervals (timesteps) assumed constant in the hiatus. Using the positions, velocities and forces of individual atoms at the current timestep, the position and velocities at the following timestep can be calculated. Forces are then recalculated, and the procedure is repeated until the desired timescale is reached.

Algorithms for classical MD simulations are implemented in a wide variety of software (NAMD, ${ }^{23}$ AMBER, ${ }^{24}$ and GROMACS, ${ }^{25}$ for example), which are compatible with a broad range of biomolecular forcefields (CHARMM, ${ }^{26} \mathrm{AMBER}^{27} \mathrm{OPLS}^{28}$ etc.) Forcefields generally differ in their functional form, parameterization protocol and parameters therein, and are generally obtained to accurately reproduce of experimental and/or quantum mechanical data. Generally, individual terms for bond lengths and angles utilize simple harmonic potentials on the basis of an energetic penalty associated with a deviation from the equilibrium value. ${ }^{26}$ To model the rotation of atoms, a torsional angle potential function is also typical to associated steric barriers. To obtain the energy of non-bonded interactions in additive forcefields, fixed-point charges are assigned at the nuclei and Coulomb and Lennard-Jones potentials are used to express electrostatic and van der Waals forces respectively. In recent years, polarizable forcefields have become available, which take into account charge polarization effects. Multiple schemes have been proposed to take into account charge fluctuations, induced by the presence of additional molecules. ${ }^{29}$ The fluctuating charge model, the induced dipole model and the Drude oscillator approach, are available via the 
CHeq forcefield, ${ }^{30,31}$ AMOEBA $^{32}$ and the CHARMM Drude polarizable forcefield, ${ }^{33,34}$ respectively. Reference [32] is recommended as an encyclopedic update of the field. ${ }^{35}$

The timescales that can be achieved by classical MD simulations are limited by the system size and availability of computing resources and efficient simulation algorithms. Simulations from 100 nanoseconds to one microsecond are generally now routine for membrane proteins systems with between 100,000 and 500,000 atoms. Simulations of this time and length scale are therefore, suitable to study a broad-spectrum of biological processes. The supercomputer Anton and optimized MD software developed by D. E. Shaw Research, has enabled production of millisecond long unbiased simulations, further extending the phenomenon that can be considered. ${ }^{36}$ In parallel, various methods have been developed to enhance sampling using typical highperformance computing facilities. In coarse-grained (CG) molecular dynamics, a reduced representation is used; by treating a group of atoms as a single interaction site, the number of degrees of freedom in a simulation system is reduced, alongside the calculation expense of each timestep. Furthermore, the timestep of each iteration is increased, facilitating the simulation of larger systems with increased complexity. Several CG forcefields have emerged in recent years, suitable for the study of biological systems. ${ }^{37-41}$

Various methodologies also exist to calculate the free-energy of relevant biological processes. Free energy perturbation (FEP) is one such method, whereby unphysical alchemical transformations are performed to convert the system between thermodynamic states; free energy differences can then be calculated using a thermodynamic cycle. ${ }^{42,43}$ FEP is predominantly used to calculate to relative binding affinities between related compounds, which is computationally inexpensive compared to calculating absolute binding affinities. ${ }^{44}$ In this context, the free energy of difference of bound and unbound states is calculated e.g. $\Delta G 1: M+L_{1} \rightarrow M_{1}$ and $\triangle G 2: M+L_{2}$ $\rightarrow \mathrm{ML}_{2}$. The relative free energy of binding can be obtained using the relation $\Delta(\Delta \mathrm{G})=\Delta \mathrm{G} 2-\Delta \mathrm{G} 1$. Several other algorithms exist which accelerate sampling along a pre-defined set of reaction coordinates, known as collective variables (CV), to overcome energetic barriers and evaluate the free-energy of the chosen conformational transition, known as the potential of mean force (PMF). Umbrella sampling (US) ${ }^{45}$ is a common enhanced sampling method in this field. To sample a specific transition, independent MD simulations are performed at energetically distinct regions of the potential energy surface, maintained by a biasing potential. These simulations, known as windows, are chosen to so that they overlap to some extent, and can then be combined and 
unbiased using the weighted histogram analysis method (WHAM) ${ }^{46,47}$ or umbrella integration to obtain the underlying potential energy surface. ${ }^{48}$

In steered molecular dynamics (SMD) simulations, an external force is applied to an atom or group of atoms, to overcome barriers and sample a specific process. ${ }^{49}$ Relative free energies can then be obtained by the Jarzynski's equality, which relates the free energy difference between two thermodynamic states and work done for the interconversion between states. ${ }^{50}$ In metadynamics, ${ }^{51}$ a bias potential is also used to accelerate sampling along chosen CVs. Gaussian functions are added to CVs, which redirects the simulation away from low-energy configurational space that has been previously inhabited. In this manner, the system departs from local freeenergy minima, exploring barrier regions and locating alternative thermodynamic states. Once the free energy profile has been flattened, convergence can be achieved, and the free energy profile can be reconstructed to provide an unbiased estimate of the landscape as a function of the CVs. The adaptive biasing force method $(A B F)^{52}$ is largely based on thermodynamic integration, whereby the instantaneous force along the reaction coordinate is evaluated directly and counteracted by an external biasing force of equal and opposite magnitude. This effectively provides a smooth energy landscape, and uniform sampling irrespective of energetic barriers allowing accelerated dynamics.

Finally, we remark upon related methods which are applicable to the identification of binding sites in macromolecules. Molecular docking is an inexpensive tool to establish potential binding sites and predict the position, orientation and binding affinities of bound molecules. This is achieved by use of sampling algorithms to catalog possible ligand-receptor conformations, which are then ranked by scoring functions. Commonplace sampling and scoring algorithms and tools to perform molecular docking are reviewed elsewhere in the literature. ${ }^{53,54}$ It should be highlighted that molecular docking is not a simulation method and can be performed on a single core-processor, or via online servers. However, molecular docking is typically performed using a rigid receptor and flexible ligand, neglecting changes in protein structure which may influence the conformation of the ligand. Therefore, the accuracy of this method is inherently limited in comparison to simulation methodologies.

"Flooding" is an alternative option, with greater computational expense and accuracy. In this case, an excessive concentration of ligand is inserted in the solution surrounding the protein target and allowed to enter favorable positions over the course of an unbiased MD simulation trajectory. ${ }^{55}$ This approach implicitly takes into account fluctuations in receptor and ligand dynamics. 


\section{Anesthetic interactions with membranes.}

Investigations into the molecular mechanisms of anesthesia have historically been fixated on the interaction of anesthetic compounds and membranes, a legacy of the Meyer-Overton correlation. In the field of computation, initial studies followed this trend, with MD simulations becoming an established tool to investigate the atomic interaction between anesthetic agents and lipid bilayers, which act as a simplified model of the plasma membrane. Unbiased all-atom MD simulations have proven successful in characterizing the effect of anesthetics on the structural properties of lipid bilayers, with coarse-grained MD simulations used in a handful of cases. ${ }^{56,57}$ The first simulation study to compare bilayer properties in the presence and absence of an anesthetic, by Huang and coworkers in 1995, inserted a single trichloroethylene molecule into a bilayer constituted of 24 DOPC (1,2-dioleoyl-sn-glycero-3-phosphocholine) molecules and an $8 \AA$ layer of explicit water molecules to solvate the system. ${ }^{58}$ Moreover, enhanced-sampling methods are commonly used to calculate the free-energy profile of anesthetics crossing model membranes. In 1996, Pohorille et al. calculated the first free-energy profiles of membrane crossing for anesthetics, using one molecule of the closely related triflouroethane across a bilayer of 72 glycerol 1-monooleate molecules solvated by $\sim 1000$ water molecules. ${ }^{59}$ In the two decades since the initial publication of these studies, the significant advancements in computer software and hardware have enabled similar simulation protocols to be performed on larger systems with increased complexity on extended timescales. In several cases, such simulations have been used in conjunction with $\mathrm{NMR}^{, 60,61}$ small-angle neutron scattering ${ }^{62}$ and $\mathrm{X}$-ray scattering ${ }^{63}$ to determine the structural attributes of model membranes. An exhaustive list of publications of membrane-anesthetic simulations, categorized by compound and bilayer composition, can be found in Table 1. As is demonstrated, neutral one-component bilayers have primarily been used, although binary mixtures including charged species in symmetric or asymmetric distributions have now been reported. ${ }^{62,64-68}$ The double bilayer system was also employed in a recent publication to impose a concentration gradient and promote permeation within unbiased MD simulations. ${ }^{69}$ In this method, two bilayers are included in different planes of the $z$ axis, to circumvent the effects of periodic boundary conditions, allowing different solute concentrations on alternate sides. Furthermore, analysis tools are now widely available, either within the simulation software itself as implemented in GROMACS, for example, ${ }^{25}$ or via additional plug-ins such as MEMBPLUGIN for VMD. ${ }^{70}$ Structural characteristics that can easily be monitored include, but are not limited to bilayer thickness, area per lipid, acyl chain segment order parameters, charge and mass density 
distribution, electrostatic potential, interdigitation fraction, water dipole orientation, hydrogen bonding and lateral pressure profiles. Here, we review a number of common themes in the literature regarding anesthetic-membrane properties.

Table 1. Survey of MD simulations studies performed of anesthetic agents and related compounds. Lipid types are abbreviated as follows: DOPC (1,2-dioleoyl-sn-glycero-3-phosphocholine), DMPC (1,2dimyristoyl-sn-glycero-3-phosphocholine), DPPC: 1,2-dipalmitoyl-sn-glycero-3-phosphocholine and POPC (1-palmitoyl-2-oleoyl-sn-glycero-3-phosphocholine).

\begin{tabular}{|c|c|c|c|c|c|c|c|}
\hline \multirow[b]{2}{*}{ Type } & \multirow[b]{2}{*}{ Name } & \multicolumn{4}{|c|}{ One-component } & \multirow{2}{*}{\multicolumn{2}{|c|}{$\begin{array}{c}\text { Binary Mixtures } \\
\text { Other }\end{array}$}} \\
\hline & & DOPC & DMPC & DPPC & POPC & & \\
\hline \multirow{10}{*}{$\begin{array}{l}\text { Local } \\
\text { Anesthetics }\end{array}$} & Articaine & & $71-73$ & & 74 & & \\
\hline & Benzocaine & & & 75 & 76 & & $64-66$ \\
\hline & Benzyl Alcohol & & 77 & & & 60 & \\
\hline & Bupivacaine & & & & 78 & & \\
\hline & Etidocaine & & & & 79 & & \\
\hline & KP-23 & & & & 80 & & \\
\hline & Lidocaine & & $61,73,81,82$ & 83 & & & \\
\hline & Prilocaine & & & & 79,84 & & \\
\hline & Procaine & & 85 & 83 & & & \\
\hline & Tetracaine & & 85 & 83 & & & \\
\hline \multirow{12}{*}{$\begin{array}{l}\text { General } \\
\text { Anesthetics }\end{array}$} & Alcohols & 86 & $61,87,88$ & 89 & & 89 & 62,67 \\
\hline & Chloroform & 68,90 & & 91-94 & & 90 & 68 \\
\hline & Diethyl Ether & & & $91,93,94$ & & & \\
\hline & Enflurane & & & $91,93,94$ & & & \\
\hline & Halothane & 95 & $63,96-98$ & $\begin{array}{l}91,93,94,9 \\
9,100\end{array}$ & 101 & 102 & \\
\hline & Desflurane & & & & 103 & & \\
\hline & Isoflurane & & & & 103,104 & & \\
\hline & Ketamine & & & & & 105 & \\
\hline & Propofol & & 106 & & 103 & & \\
\hline & Sevoflurane & & & & 103 & & \\
\hline & Trichloroethylene & 58 & & & & & \\
\hline & Xenon & 69 & & 57,107 & & 108,109 & \\
\hline $\begin{array}{l}\text { Non- } \\
\text { Immobilizers }\end{array}$ & Hexafluroethane & & 110,111 & & & 102 & \\
\hline
\end{tabular}

A number of molecules have been identified which are structurally related to anesthetic compounds but exhibit negligible anesthetic properties despite displaying similar solubilities and fulfilling the Meyer-Overton rule..$^{5,7,112}$ This has provoked considerable interest in differential responses of anesthetic and such 'non-immobilizer' molecules. Experimentally, it has been disclosed that anesthetic molecules modulate the orientational order of the hydrocarbon chains, whereas related non-immobilizer molecules produce little or no response. ${ }^{113,114}$ Observations from computation strongly agree with this postulate. Early calculations from Pohorille and colleagues of both classes of molecule in a simple lipid bilayer revealed anesthetic molecules preferentially reside near the headgroup-water interface, and non-immobilizer analogues can penetrate into the 
hydrophobic membrane interior, in non-specific locations. ${ }^{59,115,116} \mathrm{MD}$ simulations of halothane, in a saturated bilayer, confirmed this to be the case, ${ }^{99,100}$ identifying a number of perturbations in the structure of the lipid bilayer, such as an increase in area per lipid, a change in the orientation of the headgroup dipole and a decrease in the orientational order of the hydrocarbon chains, as predicted experimentally. ${ }^{113,114}$ Such changes were then absent in the presence of the halothane analogue, hexafluoroethane. ${ }^{110,111}$ These experiments were then repeated with highly unsaturated lipid bilayers, which are often present in biological membranes. ${ }^{102}$ The unsaturated systems displayed analogous distributions of compounds across the bilayer, with the exception of an additional minimum for halothane at the bilayer center and exhibited similar effects on the electrostatic profiles. Overall, the associated studies advocate that anesthetic and nonimmobilizer molecules occupy distinct locales in lipid bilayers, irrespective of the degree of chain saturation, a phenomenon which may underlie the discrepant responses to chemically similar molecules biologically.

The protonation state of individual anesthetic molecules, which affect its biological activity, must be also considered, particularly for local anesthetics. ${ }^{117}$ Comparative simulations between charged and uncharged forms of local anesthetic molecules have been reported in a number of cases, with observable differences shown. Firstly, simulations of various neutral and charged KP-23 LA molecules in neutral and charged forms were performed. ${ }^{118}$ Neutral molecules partitioned into the bilayer core whereas charged molecules self-condensed on the bilayer surface, consistent with experimental studies demonstrating differential intercalation. ${ }^{119}$ In agreement with this, charged lidocaine molecules stably occupied the lipid headgroup region in a DMPC (1,2-dimyristoyl-snglycero-3-phosphocholine) bilayer primarily oriented parallel to the bilayer normal, whereas neutral molecules can penetrate further and move more freely in a perpendicular orientation. ${ }^{81}$ In a subsequent study, the neutral and charged lidocaine molecules were found to modify the electrostatic potential profiles to a similar extent, despite alternate positions and the associated effects. It was then suggested changes of this magnitude $(90 \mathrm{mV}$ for $9 \mathrm{~mol} \%$ of lidocaine and 220 $\mathrm{mV}$ for $28 \mathrm{~mol} \%$ of lidocaine) could potentially alter the function of voltage-gated ion channels. ${ }^{82}$ Using the same simulation protocol, articaine charged and uncharged molecules were observed to accumulate in analogous positions and crossing to a similar extent as those observed in lidocaine. ${ }^{71}$ However, critical differences are observed in the orientation of the charged form, now parallel to the membrane surface, and the hydrogen bonding and diffusion attributes, resulting from an additional carbonyl group at the opposite end of the molecule, and causing differential 
effects in the membrane physical properties. The effect on the electrostatic potential profile was also similar for both forms, again of the order of the transmembrane potential. What emerges from such studies is clear evidence that charged molecules preferentially bind to the headgroup regions whereas neutral molecules penetrate the hydrophobic region of the bilayers more readily. Consequentially, the impact of anesthetic intercalation on the structural properties can differ. Efforts have also been focused towards understanding the effects of pressure on general anesthesia, in order to rationalize the known pressure reversal, i.e. the inverse correlation between pressure and anesthesic potency. ${ }^{120,121}$ Computational simulations have been used to evaluate the effect of pressure on the behaviour of anesthetic molecules, although other mechanisms of pressure modulation may exist in this context. Using halothane in artificially high concentrations, six times the clinical concentration, MD simulations by Chau et al. revealed elevated aggregation of halothane at high pressures in DMPC (1,2-dimyristoyl-sn-glycero-3phosphocholine) bilayers. ${ }^{96,98}$ The authors suggest preferred accumulation disfavors binding of individual halothane to putative binding sites on intrinsic membrane proteins. At a lesser concentration, twice the clinical concentration, and using an alternative membrane (POPC: 1palmitoyl-2-oleoyl-sn-glycero-3-phosphocholine), Tu and coworkers disclosed halothane aggregation at $2 \times 10^{7} \mathrm{~Pa}$, but not at $4 \times 10^{7} \mathrm{~Pa}$, consistent with conditions where pressure reversal is observed and inhibited. ${ }^{101}$ In contrast, such clustering of isoflurane molecules in lipid bilayers is not observed at elevated pressures, suggesting previous results may be isolated examples, and therefore an alternative mechanism may be in action. ${ }^{104}$ The question of pressure reversal will likely spawn numerous studies in the near future.

The noble gas xenon has been of considerable interest as a general anesthetic in recent years. ${ }^{122-}$ ${ }^{124}$ Although the clinical benefits of xenon have been known for decades, ${ }^{125}$ the feasibility of the gas has been newly realized with lowered costs. ${ }^{122}$ However, the mechanism of xenon remains elusive. Xenon is known to elicit a response in certain glutamatergic receptors, ${ }^{126,127}$ nicotinic receptors ${ }^{128}$ and potassium channels, ${ }^{129}$ yet is ineffective on GABAA receptors, ${ }^{126}$ a significant anesthetic target. Thus, computational efforts have been focused towards assessing the viability of the membrane-mediated mechanism of anesthetic action for xenon. The ramifications of xenon penetration on bilayer properties, pressure coupling and comparative studies of similar chemical species lacking anesthetic activities have been extensively assessed in this case.

Initial simulations of xenon in POPC (1-palmitoyl-2-oleoyl-sn-glycero-3-phosphocholine), bilayer identified slight increases in both area per lipid, membrane thickness and orientational order of 
the lipid tails, as a result of xenon occupation of the interfacial region and in the center of the bilayer. ${ }^{107}$ In a POPE bilayer, increases in area and volume per lipid and decreases in the orientational order of acyl chains were observed in the presence of xenon alongside increased diffusivity of the lipid molecules. ${ }^{109}$ The simulations conducted at a number of pressures $(0.1,10$, 20,35 , and $50 \mathrm{MPa}$ ) reveal xenon molecules are pushed into the interface between the two bilayer leaflets at high pressures which limits their diffusion. Comparable properties are observed in these bilayers and xenon-free bilayers at low pressure, advising that pressure reversal emanates from the elimination of diffusive xenon molecules in the membrane. Differential scanning calorimetry measurements combined with MD simulations confirmed favorable binding in the bilayer core, promoting straightening of the lipid tails concomitantly with increased head-group spacing. ${ }^{69}$ Biophysical properties were also perturbed, such as the decrease in phase transition temperature and the lateral pressure profile in the head group region, adhering to the lipid-bilayer mediated mechanism of general anesthesia proposed by Cantor. ${ }^{130}$ This proposal was supported further by a comparative study of four noble gases ( $\mathrm{Ne}, \mathrm{Ar}, \mathrm{Kr}$ and $\mathrm{Xe}$ ), which found that the degree of disruption of the membrane was dependent on the site of localization, and correlated well with their relative narcotic potencies. ${ }^{108}$ Booker and Sum hypothesize that the modified phase transition temperature, lateral forces and hydrophobic mismatch caused by accretion of xenon in the membrane can contribute to the conformational cycle of ion channels embedded in the membrane, providing multiple indirect mechanisms of action. ${ }^{69}$ The authors note that the suggested membrane-mediated mechanism for xenon likely contributes to an additional proteinmediated mechanism.

\section{Anesthetic interactions with ion channels.}

\subsection{Pentameric ligand-gated ion channels or "Cys-loop" receptors.}

Ion channels facilitate the passive diffusion of ionic species down their electrochemical gradient from the extracellular medium into the cell cytoplasm. Permeation is controlled by the onset of various external stimuli, such as transmembrane voltage, heat, ligand binding, and mechanical stretch, and is responsible for regulating electrical signals across the cellular membrane.

Pentameric ligand-gated ion channels ( $\mathrm{LLGICs)}$ ) are responsible for the swift conversion of chemical signals to electrical impulses throughout the nervous system. ${ }^{131}$ pLGICs from the Cys-loop receptor family have been identified as a putative target for general anesthetics at clinical concentrations, with the exact response, inhibition or potentiation), dependent on the channel type. ${ }^{132,133}$ 
Extensive work on nicotinic acetylcholine receptors revealed the overall structural assembly of pLGICs, contain five homologous or closely related heterologous subunits which assemble in a pentameric arrangement. ${ }^{134}$ Each subunit contain $\mathrm{N}$ - and C-terminal extracellular domains, and cytoplasmic and transmembrane domains. ${ }^{134}$ Orthosteric and allosteric binding sites are located in the extracellular domain, alongside the characteristic "Cys-loop", a thirteen-residue loop flanked by cysteine residues engaged in a disulfide bond. ${ }^{135}$ The symmetry axis of the transmembrane domain constitutes the ion permeation pathway, formed from four transmembrane segments (M1-M4); M2 lines the central pore and a cytoplasmic domain of variable length intersects segments $\mathrm{M} 3$ and $\mathrm{M} 4$.

The discovery of prokaryotic homologues of pLGICs hastened the publication of high-resolution crystal structures pLGICs, with pLGICs from Erwinia chrysanthemi (ELIC) ${ }^{136,137}$ and Gloebacter violaceus (GLIC), ${ }^{138,139}$ the first to become available and representing closed and open states, respectively. The structures consist of a homologous transmembrane domain (M1-M4) and an expansive extracellular domain composed of five inner and three outer strands connected by loops forming a ß-sandwich. The susceptibility of the prokaryotic homologue from Gloeobacter violaceus (GLIC) to clinically relevant concentrations of GAs has also been established. ${ }^{140}$ Therefore, such structures provide a rational study point to study the molecular mechanism of anesthetic action by computational means. Due to the number of simulation studies on the GLIC channel, and the availability of high-resolution crystal structures of the GLIC, we will focus on this channel although it should be noted that other Cys-loop receptors, such as the glycine receptor, ${ }^{141-143}$ the nicotinic acetylcholine receptor $(\mathrm{nAChR})^{144-146}$, the $\mathrm{GluCl}$ channel ${ }^{147}$ and the $\mathrm{GABA}_{A}{ }^{148-151}$ receptor, have been the subject of computational works, primarily using homology models.

Initial computational works utilizing the apo structures of GLIC aimed to identify anesthetic binding sites. ${ }^{55}$ Brannigan et al. performed a comparative study of isoflurane binding to $\mathrm{nAChR}$ and GLIC, implicating eight distinct sites, throughout the transmembrane and extracellular domains, and within the interfacial region between the two. ${ }^{55}$ Of potential functional relevance, the pore of both LGICs accommodates isoflurane dimers which would impose a physical block to ion permeation, thus acting as an inhibitory site. Isoflurane also binds to the TM domain of nAChR in intersubunit sites underneath the M2-M3 loop in $\mathrm{AAChR}$, in agreement with earlier speculation from photoaffinity labelling. ${ }^{152}$ The absence of such a site in GLIC leads the authors to postulate this acts as a positively modulating site, rationalizing the increased sensitivity of GLIC to inhibition 
by anesthetics, relative to nAChR. ${ }^{140}$ Finally, novel intrasubunit sites in both $\alpha$ nAChR subunits, and one $\alpha$ GLIC subunit, were observed. It is speculated that residence of the $\alpha$ subunits, which determine agonist susceptibility, in a position behind the $\mathrm{M} 2$ helices, which are involved in channel opening, and parallel to the hydrophobic constriction, could influence the active state of the receptor.

Various crystal structures have since been resolved of GLIC in complex with anesthetic molecules, including propofol, ${ }^{153,154}$ desflurane, ${ }^{153}$ bromoform, ${ }^{154-156}$ ethanol ${ }^{155}$ and ketamine, ${ }^{137}$ providing additional insights into sites in the transmembrane and extracellular domains, and in the pore (Figure 1). A transmembrane anesthetic binding site was characterized in the 2011 structure of GLIC in complex with GAs propofol and desflurane. ${ }^{153}$ Here, the compounds occupy an intrasubunit crevice in the extracellular leaflet of the transmembrane domain, with residues from M1-M4 and $\beta 6-\beta 7$ loop of the extracellular domain. Mutants T255A (M3) and V242M (M2) displayed a marked, albeit distinct, response to propofol and desflurane, confirming the physiological importance of such a site. Increased propofol sensitivity was interpreted by MD simulations within the same study, as propofol percolated further into the cavity, becoming less mobile and intensifying interactions with residue 242 in mutant channels relative to the wild type.

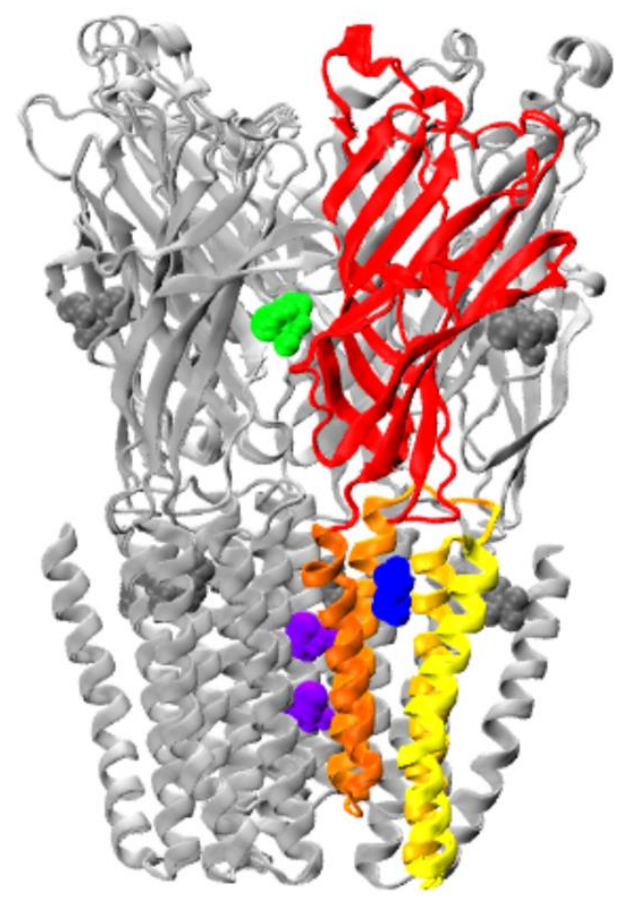

Figure 1. Position of propofol (blue), isoflurane (purple) and ketamine (green) binding sites in GLIC/ELIC obtained from PDB files 3P50, ${ }^{153} 4 \mathrm{Z90} 0^{157}$ and $4 \mathrm{~F} 8 \mathrm{H},{ }^{158}$ respectively. Transmembrane helices of a single subunit are colored yellow to orange, with the extra cellular domain shown in 
red.

Using the crystallographic positions of desflurane, as a reference, and additional molecular docking calculations, Willenbring and coworkers simulated isoflurane binding to GLIC to delineate the structural consequences of prolonged binding. ${ }^{159}$ Movement of isoflurane between sites promoted the breakage of intersubunit salt bridges in the extracellular domain, perturbing the quaternary structure and inward movement of the $M 2$ helices, providing a mechanism by which isoflurane contributes to channel closure.

Subsequent MD simulations revealed loose binding of the resolved desflurane to the crystal structure site, which could dissociate in under 100 ns. ${ }^{160}$ However, occupation of an adjacent site enhanced binding suggesting double occupation is required for desflurane to inhibit GLIC. Mutation in this site experimentally correlated with diminished binding in the crystal structure site, in agreement with this. The route of access of such sites is also exposed, with desflurane diffusing into and out of the site through the membrane, via the protein-membrane interfacial region.

Further to this, fluorescence quenching disclosed the presence of multiple binding sites for inhaled anesthetic halothane and the intravenous anesthetic thiopental in the transmembrane domain. ${ }^{161}$ Simulations revealed a site in close proximity to N200, near M1 and the M2-3 loop, which was confirmed by mutagenesis of this residue, and multiple additional sites. Some sites overlapped to some extent for the chemically distinct compounds and could therefore quench the same tryptophan residues. Halothane bound in the M1-M23 site stabilized the M23 loop whilst mobilizing the Cys-loop in the EC domain, two regions implicated in channel gating in pLGICs. ${ }^{162-164}$ Binding of halothane near W160 at the EC-TM disrupted critical salt bridges, such as D32R192. ${ }^{161,165}$ Removal of the analogous salt bridges in other ligand-gated ion channels notably diminished the stability of the open state ${ }^{164,166,167}$ suggesting occupation of this site may exert a similar effect in GLIC.

Transmembrane binding was also observed for propofol via flooding simulations using coarsegrained methods which accessed a multi-microsecond timescale. ${ }^{168} \mathrm{GLIC}$ channel dynamics with partial occupancy of propofol binding site was investigated by Mowrey et al. ${ }^{169}$ Asymmetric states with one to three sites inhabited exhibited elevated channel dehydration relative to symmetric states with zero or five molecules bound. Conformational changes were observed in the 
asymmetric states, consistent with the known effects of asymmetric agonist binding in homopLGICs. ${ }^{170-172}$

With regards to a potential pore-binding site, crystal structures of GLIC bound to bromo-lidocaine, a lidocaine analogue provided the first structural insights into a pore-binding site in pLGICs. ${ }^{173}$ Computationally, isoflurane was also documented to pass through the closed hydrophobic gate, ${ }^{159}$ in spite of dehydration of the central cavity, occupying a position between residues 1233 and I240, supporting earlier theoretical and experimental evidence of pore-binding in GLIC. ${ }^{55,173}$ LeBard et el. performed a systematic study of binding to the GLIC pore, ${ }^{174}$ using monomers and dimers of both isoflurane and propofol, in addition to ethanol as a negative control. Isoflurane binding affinities, calculated using FEP, were contingent on the functional state of the pore, with a situation involving binding of two isoflurane molecules to the closed pore resembling the micromolar affinity determined experimentally, and consistent with a pore-block mechanism. The determined affinities illustrated that the effect of propofol is overestimated when considering a pore-block mechanism in isolation and support a mechanism where pore and allosteric sites exert competitive effects on the functional state of the channel. ${ }^{175}$

These conclusions were further supported by the high-resolution structure of ELIC co-crystallised with isoflurane, in both apo and agonist bound forms, presumed to be in resting and desensitized activation states, respectively. ${ }^{157}$ These structures show inhabitancy of two isoflurane molecules within the pore, with MD simulations in the same study revealing paramount stability in the ELIC resting state, as a result of observable changes in the pore radii along the channel. Nevertheless, the affinity for both states suggests isoflurane can act by stabilizing the closed channel and also obstructing the open channel. The structural and dynamical information presented throughout these works strongly supports a pore-binding mechanism of inhibition. Bromoform binding has also been observed in the pore of GLIC, experimentally and computationally. ${ }^{155,156}$

Finally, structural information has also revealed a binding site in the extracellular domain. The crystal structure of $R$-ketamine in complex with GLIC exhibits symmetric binding in extracellular intersubunit cavities. ${ }^{137}$ MD simulations confirmed the observed stereospecifity of this site, with $S$ ketamine unable to interact with residue D154, which stably interacts with $R$-ketamine. ${ }^{176}$ Notably, $R$-ketamine evolved into asymmetric binding poses over the course of the trajectories, in contrast to the known symmetric structures of pLGICs in complex with anesthetics. ${ }^{137,153,155}$ Structural differences resulting from $R$-ketamine binding was propagated to the ECD-TMD interface and to 
pore-lining helix $M 2$, via either the pre-M1 region or the EC $\beta 1-\beta 2$ loop. ${ }^{176,177}$ In coarse-grained simulations by Joseph and Mincer, mentioned previously, propofol spontaneously occupied the crystallographic extracellular binding site, prompting asymmetric conformational changes in GLIC, transmitted through a similar allosteric network in the ECD-TMD interface. ${ }^{168}$ The molecular mechanism of long-range allosteric coupling was further probed by coarse-grained MD simulations, using a novel elastic network model proposed by Li and colleagues. ${ }^{178}$

Overall, a myriad of anesthetic binding sites have been proposed by both experimental and simulation methods for the GLIC channel. Consequently, uncovering the molecular mechanism of anesthetic action has proven difficult. Several studies of GLIC with a number of different anesthetics reported a degree of structural asymmetry during the inhibition of GLIC by anesthetics.

\subsection{Voltage-gated ion channels}

The voltage-gated ion channel (VGIC) family that specifically conduct $\mathrm{Na}^{+}(\mathrm{Nav})$ and $\mathrm{K}^{+}$ions (KV) implements critical actions in the generation and propagation of action potentials, and thus, plays a crucial physiological role. VGIC's are a common target for antiarrhythmic agents, local anesthetics, anticonvulsants and pain therapeutics. ${ }^{179,180}$ Anesthetics are known to block both Nav and $K_{v}$ currents at clinically relevant concentrations. ${ }^{181,182}$ Various computational studies have been performed to investigate this phenomenon on a molecular level.

The first high-resolution structure of the pore domain of an ion channel, the KcsA channel from Streptomyces lividans, was revealed in $1998 .{ }^{183}$ The protein is characterized by a symmetric tetrameric arrangement with each monomer containing two transmembrane $\alpha$-helices and an intermittent pore loop. The pore loop contains the signature selectivity sequence, which determines the ionic permeability of the channel, and thus has become known as the selectivity filter. ${ }^{184,185}$ Between the selectivity filter and the cytoplasm, a water-filled cavity is present to provide an ideal environment for ion transfer. ${ }^{186} \mathrm{In} \mathrm{K} \mathrm{K}^{+}$channels, the selectivity filter is constituted of a conserved amino acid sequence arranged to form rings of oxygen atoms, which are able to bind dehydrated ions in a cage-like structure, in adjoining sites. In $\mathrm{Na}^{+}$channels, a ring of residues at the extracellular site forms the selectivity filter, although the identity of residues is more dependent on the specific channel. Atomistic structures of bacterial (KvAP from Aeropyrum pernix) ${ }^{187,188}$ and mammalian (Kv1.2) ${ }^{189-192} \mathrm{~K}_{\mathrm{V}}$ channels have since been obtained, illuminating the structure of voltage-sensor domains (VSD) and their interaction with the pore domain. In contrast, high-resolution crystallographic data of Nav channels has been only available since 2011 from a 
number of bacterial sources: NavAb (Arcobacter butzleri), ${ }^{193-196}$ NavRh (Rickettsiales sp. HIMB114), ${ }^{197,198}$ NavMs (Magnetococcus sp.) ${ }^{199-201}$ and NavAe1p (Alkalilimnicola ehrlichii sp. nov. $)^{202}$. These channels exhibit four identical subunits arranged symmetrically, each containing six transmembrane segments (S1-S6). S1-S4 constitute the voltage-sensing domain, whilst S5-S6 segments represent the pore domain, described previously. The behaviour of the voltage sensor and pore domains are closely coupled, whereby movement of S4 (following a change in the transmembrane voltage) prompts rearrangement of the pore helices, via the S4-S5 linker, subsequently opening the cytoplasmic gate. Recently, a cryo-EM structure of a eukaryotic Nav channel, NavPs, at near-atomic resolution (3.8 $)$ ), isolated from the American cockroach Periplaneta Americana has been published. ${ }^{203}$ This channel is comprised of a single polypeptide chain which is arranged into equivalent homologous transmembrane domains, as is the case for eukaryotic Nav channels. The overall homology observed between known eukaryotic and prokaryotic structures advocates bacterial analogs are suitable templates to study small-molecule inhibition of $\mathrm{K}_{v}$ and $\mathrm{Nav}_{\mathrm{v}}$ channels.

\subsubsection{Sodium Channels}

The therapeutic value of Nav channel inhibitors is well-established, with antiarrhythmics, anticonvulsants, antidepressants and antiepileptics known to block the Nav channel pore. Various anesthetic agents, including but not limited to isoflurane, ${ }^{204,205}$ lidocaine ${ }^{206}$ and bupivacaine ${ }^{206}$ are also known to modulate the entire mammalian Nav channel family. The effect of local anesthetics on sodium currents was first acknowledged by Weidman in $1955 .{ }^{207}$ This idea was matured by various researchers into the 1970's, leading to the proposal of several mechanisms of anesthetic action on $\mathrm{Na}_{v}$ channels. ${ }^{208}$ Making use of data on $\mathrm{Nav}$ channel blockage by quarternary derivatives of lidocaine in myelinated nerves, Strichartz established the concepts of "tonic" and "usedependent" block, whereby drug molecules specifically bind to the closed and open state of the channel, respectively. ${ }^{209}$ At this time, factors including the structure/protonation state of the molecule, the mode of application and the membrane potential were found to alter the exact contribution of each mechanism to the observable response of individual anesthetics. ${ }^{210}$ Nowadays, it is widely accepted that highly polar or charged compounds are effective during usedependent block traversing through a hydrophilic pathway, whereas neutral compounds are effective during tonic block, passing through a hydrophobic pathway when applied from either side of the membrane. ${ }^{208,211}$ 
The question of an alternative hydrophobic pathway was answered to some extent by the available structural information, which revealed lateral lipid-filled openings, termed fenestrations, directly connecting the central pore to the surrounding membrane, illustrated in Figure 2. Considering this, computational methods have been used to investigate small-molecule binding sites in Nav channels and potential access pathways for those within the pore.

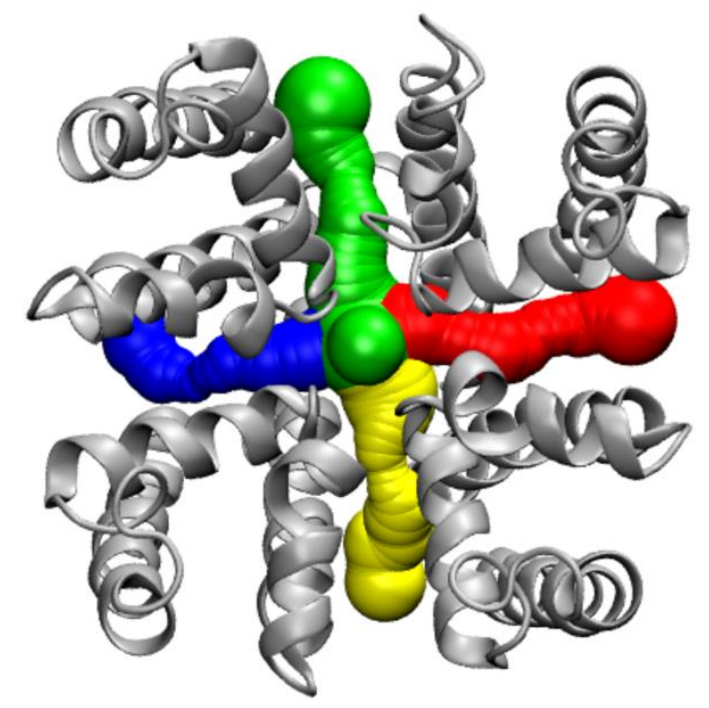

Figure 2. Pore structure of the NavMs channel, with the individual fenestrations colored in blue, red, yellow and green. Each of the four monomers is represented in grey. The view of the channel is perpendicular to the membrane plane.

The first prokaryotic Nav channel to exhibit a response to anesthetics, ${ }^{212} \mathrm{NaChBac}$, has been the subject of several computational works. ${ }^{213,214}$ Homology models of NaChBac, based on a NavAb structure (PDB ID: 3RVY), in combination with flooding simulations, were used to identify multiple binding sites for the general anaesthetics isoflurane and sevoflurane. ${ }^{213,214}$ Sites were located near the selectivity filter accessible from the extracellular solution, at the S4-S5 linker, in the central cavity and at the entrance to the fenestration regions. The isoflurane sites with estimated binding affinities of the isoflurane interaction sites were calculated to be in the physiologically relevant range using $\mathrm{FEP},{ }^{213}$ with those beneath the selectivity filter and near the S4-S5 linker were later confirmed by NMR data. ${ }^{215}$ Evidence for state-dependent binding of sevoflurane to the S4-S5 linker and selectivity filter site was also presented in the study by Barber and coworkers, alongside a unique site in the deactivated state where the S6 helices coalesce. ${ }^{214}$ The specific locale of the observed isoflurane and sevoflurane binding sites suggests that GAs may act by interfering with the selectivity filter, activation at the cytoplasmic $\mathbf{S 6}$ gate or by obstructing ion permeation within the pore, in a similar manner to local anesthetics in the latter case. ${ }^{213}$ Isoflurane and sevoflurane molecules were able to enter the central cavity via fenestrations, bypassing the closed cytoplasmic 
gate. Entrance of such molecules was accompanied by displacement of fenestration-bound lipid molecules, the expulsion of water molecules through the selectivity filter, with approximately six water molecules displaced by a single isoflurane in the pore. These observations demonstrated the capability of fenestrations to act as a hydrophobic pathway for small drugs such as isoflurane. ${ }^{213}$

Lending further support to this postulate, Kaczmarski and Corry used unbiased MD simulations to analyze the behaviour of fenestrations on the subunit-interface between S5 and S6 helices in six bacterial Nav channels and a homology model of Nav1.4, observed. ${ }^{216}$ The bottleneck radii of the fenestrations range in size from 1.6 to $2.2 \AA$, with a maximum radii between 2.6 to $2.8 \AA$, potentially tolerable for the passage of aromatic rings, a typical component of $\mathrm{Nav}$ channel inhibitors. Fenestration size was determined by the identity (F203 in NavAb, whereas the equivalent positions in NavRh and NavMs is occupied by lle and Met) and rotameric state of bottleneck residues and the extent of lipid occupation, with local fluctuations typically observed on a nanosecond timescale. Following this, fenestration characteristics in the Nav1.4 channel model varied as a result of the sequential differences of the repeat sequences.

The location of small molecule binding has also been of interest, utilizing the high-resolution structure of bacterial channels. Studies from independent research groups have identified two high-affinity binding sites for small neutral drugs, the local anesthetic benzocaine and the antiepileptic phenytoin, in the central cavity of NavAb, using unbiased and metadynamics simulations. ${ }^{217}$ The observed binding occurs in two hydrophobic sites in the pore, in close proximity to the $\mathrm{S} 6$ gate and in a fenestration site, consistent with residues recognized in blocking of eukaryotic channels. ${ }^{218}$ Using extensive equilibrium simulations, Boiteux and coworkers cite enhanced stability of the fenestration site as a result of $\pi$-stacking with $\mathrm{F} 203$; on the other hand, Martin and Corry advocate non-specific hydrophobic interactions dominate binding in such sites, with the $\mathrm{S} 6$ site deemed more energetically favorable (by $3 \mathrm{kcal} / \mathrm{mol}$ ) using metadynamics simulations. These results are in agreement with prior studies implicating multiple residues which are hydrophobic in nature in tonic block, ${ }^{219}$ which is in stark contrast to use-dependent blockers which rely on cationic interactions with aromatic residues. ${ }^{219}$ Spontaneous translocation of benzocaine through the fenestrations is observed in the unbiased simulations, with low energy barriers of this route calculated by enhanced-sampling methods, confirming the fenestrations as a viable access route for the local anesthetic benzocaine. Unexpectedly, transfer of compounds through the closed cytoplasmic gate was noted to be viable in both studies. To imitate the 
eukaryotic Nav channel pore, aromatic residues were introduced into the NavAb pore near the activation gate, pushing benzocaine and phenytoin towards the fenestration site whereas aromatic residues near the fenestrations imposed the opposite effect. Interestingly, the binding affinity increased in such channels. ${ }^{220}$

In the NAvMs channel, Buyan et al. characterized the transport and binding properties of a selection of physiologically active compounds, permanently neutral (benzocaine, lamotrigine, and carbamazepine) and protonatable (PF-5215786, PF-6305591, and lidocaine) in both neutral and charged states at $\mathrm{pH} 7$, using unbiased MD simulations and the replica exchange with solute scaling/tempering method. ${ }^{221}$ Protonatable compounds penetrated the bilayer with ease in the neutral form, relative to their charged protonated form, advocating such molecules relinquish their charge before entering the plasma membrane. Once within the lumen of NaVMs, binding of neutral and charged molecules manifests at separate sites: neutral compounds primarily occupied the lower region of the pore proximal to $\mathbf{S 6}$ residues, whilst charged compounds displayed prominent interactions with the selectivity filter. In the latter case, sodium currents were hampered by steric block and electrostatic repulsion with the bound molecule. This novel, poreblocking site correctly reproduces the position of the resolved bromines in the crystallographic structure of NavMs with antagonist PF-5215786, ${ }^{200}$ and suggests compounds reclaim their charge to exert an effect in the pore.

\subsubsection{Potassium Channels}

Both voltage-dependent and voltage-independent $\mathrm{K}^{+}$-channels can be blocked by local anesthetics. Responses have been recorded for members of the two-pore domain 222 and inward rectifying channels. ${ }^{223}$ An initial MD study of open and closed conformations of KirBac1.1 in the presence of halothane molecules demonstrated accumulation of halothane molecules at the cytoplasmic gate, in the open state only. ${ }^{224}$ Occupation of this region dampened movements of phenylalanine residues, which have previously been implicated in channel gating. ${ }^{225} \mathrm{~A}$ similar mechanism whereby halothane moderates tryptophan fluctuations has been proposed by fluorescence experiments ${ }^{226}$ and MD simulations alike. ${ }^{227,228}$

The potential role of Kv channels in the action of inhalational general anesthetics has been realized most recently, ${ }^{229}$ with evidence accumulating from the 1990's. ${ }^{230-234} \mathrm{~A}$ direct link between the application of isoflurane and the single-channel properties of Drosophila Shaker B Kv channel was first established by Li and Correa. ${ }^{231}$ Inhibitory responses to general anesthetics (chloroform, halothane, isoflurane, and propofol) and short-chain alcohols have also been recorded for K- 
Shaw2, a distinct member of the Drosophila Shaker Kv channel family. ${ }^{235-237}$ Distinctively, sevoflurane triggered activation of K-Shaw2. ${ }^{238}$ Contrary to typical $\mathrm{K}^{+}$channels, K-Shaw2 is thought to undergo a simple activation cycle, involving only two ${ }^{239,240}$ or three states. ${ }^{238}$ Consolidation of the accrued data advocate inhibitory compounds sustain closed states of the $\mathrm{K}^{+}$channel, whereas sevoflurane is proposed to eradicate unstable inactivated states which diverge from the activation pathway. ${ }^{238}$

In order to examine pore-binding sites in voltage-dependent channels, MD simulations of the open state $\mathrm{Kv} 1.2^{190,191}$ and $\mathrm{K}_{\mathrm{Ca}} 3.1$ (a homology model using the Kv1.2 structure) were performed with local anesthetic lidocaine and TRAM-34 initially inserted in the pore. ${ }^{241}$ Both compounds occupy a conserved valine-alanine-valine motif in the pore in both channels, in close proximity to the selectivity filter, in agreement with interaction sites predicted for small molecule binding in other $\mathrm{K}^{+}$channels. ${ }^{242,243} \mathrm{Kd}$ values were estimated as $20 \mu \mathrm{M}$ (lidocaine-Kv1.2), $540 \mathrm{nM}$ (TRAM-34-Kv1.2), and $10 \mathrm{nM}$ (TRAM-34-KCa3.1), in broad agreement with experimentally determined values, and the recorded $\sim 200$-fold selectivity of TRAM-34 for KCa3.1 over Kv1.2. ${ }^{244}$ The PMF profiles of ion permeation through Kv1.2 were then calculated, in order to delineate the functional consequence of pore binding to this site. The neutral form of lidocaine had negligible effect on the calculated energetics, whilst the charged form imposed an energy barrier of the order of $6 \mathrm{kT}$, consistent with previous reports. ${ }^{245}$ On the other hand, TRAM-34 prompted ion dehydration, inducing an energy barrier of $\sim 10 \mathrm{kT}$. As a consequence, lidocaine must be neutral to cross the membrane and enter the pore yet be charged to exert an effect. In contrast, the neutral form of TRAM-34 is sufficient for both purposes, providing a potential scaffold for drugs which would remain active in acidic conditions typical of inflammation.

Various experimental results have also cited the involvement of the S4-S5 linker and the distal part of the $\mathrm{S} 6$ segment, and adjacent helices in the inhibition of K-Shaw2 by general anesthetics. ${ }^{236,237,246,247}$ The region is critical for coupling between the voltage-sensor and pore domains in the event of voltage activation, and it is therefore possible that anesthetic binding interferes with this phenomenon. ${ }^{191,248}$ In order to provide a molecular description of this phenomenon, Barber and colleagues performed a systematic alanine/valine mutagenesis scan to the S4-S5 linker and a segment of S6 on K shaw2, with associated electrophysiological experiments to delineate the consequences of such mutations on K-Shaw2 inhibition by 1-butanol and halothane. ${ }^{249}$ In conjunction with docking calculations and MD simulations, a putative anesthetic binding site in this region was identified in the S4-S5 linker region, with residues critical for either 
anesthetic binding, allosteric coupling to the binding site and propagating structural changes to the cytoplasmic gate determined. A molecular picture of closely associated, yet distinct binding and effector sites emerged, beginning to unravel the determinants of anesthetic action to $\mathrm{K}$ Shaw2.

Following this, the differential effects of the several general anesthetics and the novel potentiator sevoflurane on Kv1.2 and Kv1.2-FRAKT chimera (where the S4-S5 linker was replaced with that from K-Shaw2) were characterized. ${ }^{250}$ The output of such experiments demonstrated that discrete substitutions in the S4-S5 can induce startling differences in the response of Kv channels to general anesthetics. More specifically, positive modulation of the Kv1.2 channel was predominantly dependent on a single mutation, G329T, with the mutant response closely resembling that displayed by K-Shaw2. Docking and free-energy methods utilizing closed/resting and open/activated states, ${ }^{251,252}$ identified four sites in the wild-type and G329T mutant, two involving the S4-S5 linker, one at the S5-S6 helical interface and one in close proximity to selectivity filter. ${ }^{250}$ The selectivity filter sites displayed the highest affinity binding, and a single site at the S4-S5 linker displays noticeable dependence on the active state. Identification of several sites in the linker region with low binding affinities is consistent with a multi-site model for positive allosteric modulation of $K_{v}$ channels by sevoflurane. Further evidence was provided on this subject by Stock et al., who proposed a novel theoretical framework to study concentration-dependent interactions of ligands to multiple saturable sites in membrane receptors, based on docking and FEP calculations. ${ }^{253}$

\section{Outlook}

The molecular mechanisms of anesthesia have been the source of significant debate since the turn of the twentieth century. The Meyer-Overton hypothesis suggested anesthetic agents primarily act via the lipid membrane. However, various datum have been interpreted to disprove it, motivating alternative postulates. The evolution of computational protocols over the last two decades has permitted the study of both membrane and protein-mediated theories via molecular dynamics simulations, revealing dynamic characteristics of relevant biological processes at atomic resolution and applicable timescales.

Several lines of inquiry have provided notable success, such as the assessment of the modification of structural properties of lipid bilayers following anesthetic exposure, and the identification of anesthetic binding sites on intrinsic membrane proteins, most notably pentameric ligand-gated ion 
channels and voltage-gated cation channels. High-resolution structural information of bacterial analogs has proven indispensable in this case, providing a practical starting point for computational works. Concurrently, the publication of structures of pharmacologically relevant membrane proteins in the future will provide additional opportunities for researchers to perform simulation studies of this nature, for instance, in transient receptor potential channels. ${ }^{254}$ Substantial advancements in high-performance computing facilities, simulation algorithms and forcefields required for the study of model membrane systems have also significantly contributed to the success of this field. Further improvements in computer hardware will likely be achieved at an unprecedented rate, continuously improving the timescales that can be reached by simulation methods.

Several pertinent questions remain to be addressed. Exactly how direct and indirect effects influence the activation state of known targets, and hence evoke a physiological response has proven difficult to discern. Furthermore, detailed information regarding the subtype selectivity of known anesthetic compounds could be exploited to optimize compounds in the design of novel anesthetic compounds. It is likely that models accounting for the combined effect of smallmolecule binding and membrane perturbations will emerge in the near future.

Acknowledgments. This work was supported by the Biotechnology and Biological Sciences Research Council and Pfizer Neusentis through a CASE studentship to Victoria Oakes. This work was supported by the Royal Society through a Newton award.

\section{Biographies}

Victoria Oakes received her MChem in Chemistry from the University of Sheffield (UK) in 2014. In 2014, she joined the Domene group at King's College London and subsequently at the University of Bath to complete her Ph.D. in the modelling and simulation of membrane proteins. She was awarded a BBSRC-CASE studentship supported by Pfizer Neusentis.

Carmen Domene received her MChem in Chemistry from the University of Seville (Spain) and a Ph.D. in Chemistry from the University of Exeter (UK) where she worked with Patrick Fowler and Paul Madden on modeling many-body effects in interionic interactions. She then moved to the University of Oxford as a postdoctoral fellow at the Laboratory of Molecular Biophysics, working on computer simulations of ion channels. From 2003-2011, she held a Royal Society University Research fellowship at the Physical and Theoretical Chemistry Laboratory of the University of Oxford, and she became a Reader in Computational Chemistry at King's College London in 2012. 
From 2017, she is a full professor at the University of Bath (UK). Her current research involves probing the structure and dynamics of biomolecular systems using computer simulation techniques; systems of interest include enzymes, model membranes, and membrane-bound proteins.

\section{References}

(1) Meyer, H. Zur Theorie Der Alkoholnarkose. Archiv für experimentelle Pathologie und Pharmakologie 1899, 42, 109-118.

(2) Overton, C. E. Studien ÜBer Die Narkose Zugleich Ein Beitrag Zur Allgemeinen Pharmakologie: Jena, 1901.

(3) Taheri, S.; Halsey, M. J.; Liu, J.; Eger, E. I.; Koblin, D. D.; Laster, M. J. What Solvent Best Represents the Site of Action of Inhaled Anesthetics in Humans, Rats, and Dogs. Anesthesia and Analgesia 1991, 72, 627-634.

(4) Johansson, J. S.; Zou, H. Partitioning of Four Modern Volatile General Anesthetics into Solvents That Model Buried Amino Acid Side-Chains. Biophysical Chemistry 1999, 79, $107-$ 116.

(5) Koblin, D. D.; Chortkoff, B. S.; Laster, M. J.; Eger, E. I.; Halsey, M. J.; Ionescu, P. Polyhalogenated and Perfluorinated Compounds That Disobey the Meyer-Overton Hypothesis. Anesthesia and Analgesia 1994, 79, 1043-1048.

(6) Koblin, D. D.; Chortkoff, B. S.; Laster, M. J.; Eger, E. I.; Halsey, M. J.; Ionescu, P. Nonanesthetic Polyhalogenated Alkanes and Deviations from the Meyer-Overton Hypothesis. Progress in Anesthetic Mechanism, Vol 3, Special Issue, 1995: Proceedings of the International Workshop on Anesthetic Mechanisms 1995, 451-456.

(7) Fang, Z. X.; Laster, M. J.; Ionescu, P.; Koblin, D. D.; Sonner, J.; Eger, E. I.; Halsey, M. J. Effects of Inhaled Nonimmobilizer, Proconvulsant Compounds on Desflurane Minimum Alveolar Anesthetic Concentration in Rats. Anesthesia and Analgesia 1997, 85, 1149-1153.

(8) Pohorille, A.; Wilson, M. A.; New, M. H.; Chipot, C. Concentrations of Anesthetics across the Water-Membrane Interface; the Meyer-Overton Hypothesis Revisited. Toxicol Lett 1998, 100-101, 421-430.

(9) Cantor, R. S. Lipid Composition and the Lateral Pressure Profile in Bilayers. Biophysical Journal 1999, 76, 2625-2639.

(10) Cantor, R. S. The Influence of Membrane Lateral Pressures on Simple Geometric Models of Protein Conformational Equilibria. Chemistry and Physics of Lipids 1999, 101, 45-56.

(11) Cantor, R. S. Lateral Pressures in Cell Membranes: A Mechanism for Modulation of Protein Function. Journal of Physical Chemistry B 1997, 101, 1723-1725.

(12) Cantor, R. S. Breaking the Meyer-Overton Rule: Predicted Effects of Varying Stiffness and Interfacial Activity on the Intrinsic Potency of Anesthetics. Biophysical Journal 2001, 80, 2284-2297.

(13) Moss, G. W. J.; Lieb, W. R.; Franks, N. P. Anesthetic Inhibition of Firefly Luciferase, a Protein Model for General-Anesthesia, Does Not Exhibit Pressure Reversal. Biophysical Journal 1991, 60, 1309-1314. 
(14) Franks, N. P.; Jenkins, A.; Conti, E.; Lieb, W. R.; Brick, P. Structural Basis for the Inhibition of Firefly Luciferase by a General Anesthetic. Biophysical Journal 1998, 75, 2205-2211.

(15) Franks, N. P.; Lieb, W. R. Do General Anaesthetics Act by Competitive Binding to Specific Receptors? Nature 1984, 310, 599-601.

(16) Hemmings, H. C.; Adamo, A. I. B. Effects of Halothane and Propofol on Purified Brain Protein-Kinase-C Activation. Anesthesiology 1994, 81, 147-155.

(17) Gomez, R. S.; Guatimosim, C.; Gomez, M. V. Mechanism of Action of Volatile Anesthetics: Role of Protein Kinase C. Cellular and Molecular Neurobiology 2003, 23, 877-885.

(18) Lugli, A. K.; Yost, C. S.; Kindler, C. H. Anaesthetic Mechanisms: Update on the Challenge of Unravelling the Mystery of Anaesthesia. European Journal of Anaesthesiology 2009, 26, 807-820.

(19) Eckenhoff, R. G.; Johansson, J. S. Molecular Interactions between Inhaled Anesthetics and Proteins. Pharmacological Reviews 1997, 49, 343-367.

(20) Leach, A. Molecular Modelling : Principles and Applications; Pearson Prentice Hall, 2009.

(21) Cramer, C. J. Essentials of Computational Chemistry: Theories and Models (2nd Edition); John Wiley \& Sons, 2004.

(22) Various Computational Biophysics of Membrane Proteins (Rsc Publishing); The Royal Society of Chemistry: UK, 2016.

(23) Phillips, J. C.; Braun, R.; Wang, W.; Gumbart, J.; Tajkhorshid, E.; Villa, E.; Chipot, C.; Skeel, R. D.; Kale, L.; Schulten, K. Scalable Molecular Dynamics with Namd. J Comput Chem 2005, 26, 1781-1802.

(24) Case, D. A.; Cheatham, T. E., 3rd; Darden, T.; Gohlke, H.; Luo, R.; Merz, K. M., Jr.; Onufriev, A.; Simmerling, C.; Wang, B.; Woods, R. J. The Amber Biomolecular Simulation Programs. J Comput Chem 2005, 26, 1668-1688.

(25) Van Der Spoel, D.; Lindahl, E.; Hess, B.; Groenhof, G.; Mark, A. E.; Berendsen, H. J. Gromacs: Fast, Flexible, and Free. J Comput Chem 2005, 26, 1701-1718.

(26) Brooks, B. R.; Bruccoleri, R. E.; Olafson, B. D.; States, D. J.; Swaminathan, S.; Karplus, M. Charmm: A Program for Macromolecular Energy, Minimization, and Dynamics Calculations. Journal of Computational Chemistry 1983, 4, 187-217.

(27) Cornell, W. D.; Cieplak, P.; Bayly, C. I.; Gould, I. R.; Merz, K. M.; Ferguson, D. M.; Spellmeyer, D. C.; Fox, T.; Caldwell, J. W.; Kollman, P. A. A Second Generation Force Field for the Simulation of Proteins, Nucleic Acids, and Organic Molecules. Journal of the American Chemical Society 1995, 117, 5179-5197.

(28) Jorgensen, W. L.; Tirado-Rives, J. The Opls [Optimized Potentials for Liquid Simulations] Potential Functions for Proteins, Energy Minimizations for Crystals of Cyclic Peptides and Crambin. Journal of the American Chemical Society 1988, 110, 1657-1666.

(29) Vorobyov, I. V.; Anisimov, V. M.; MacKerell, A. D. Polarizable Empirical Force Field for Alkanes Based on the Classical Drude Oscillator Model. The Journal of Physical Chemistry $B$ 2005, 109, 18988-18999.

(30) Patel, S.; Brooks, C. L. Charmm Fluctuating Charge Force Field for Proteins: I Parameterization and Application to Bulk Organic Liquid Simulations. Journal of Computational Chemistry 2004, 25, 1-16. 
(31) Patel, S.; Mackerell, A. D., Jr.; Brooks, C. L., 3rd. Charmm Fluctuating Charge Force Field for Proteins: li Protein/Solvent Properties from Molecular Dynamics Simulations Using a Nonadditive Electrostatic Model. J Comput Chem 2004, 25, 1504-1514.

(32) Shi, Y.; Xia, Z.; Zhang, J.; Best, R.; Wu, C.; Ponder, J. W.; Ren, P. Polarizable Atomic Multipole-Based Amoeba Force Field for Proteins. Journal of Chemical Theory and Computation 2013, 9, 4046-4063.

(33) Lopes, P. E. M.; Huang, J.; Shim, J.; Luo, Y.; Li, H.; Roux, B.; MacKerell, A. D. Polarizable Force Field for Peptides and Proteins Based on the Classical Drude Oscillator. Journal of Chemical Theory and Computation 2013, 9, 5430-5449.

(34) Savelyev, A.; MacKerell, A. D., Jr. All-Atom Polarizable Force Field for DNA Based on the Classical Drude Oscillator Model. J Comput Chem 2014, 35, 1219-1239.

(35) Lemkul, J. A.; Huang, J.; Roux, B.; MacKerell, A. D. An Empirical Polarizable Force Field Based on the Classical Drude Oscillator Model: Development History and Recent Applications. Chemical Reviews 2016, DOI:10.1021/acs.chemrev.5b00505 10.1021/acs.chemrev.5b00505.

(36) Shaw, D. E.; Maragakis, P.; Lindorff-Larsen, K.; Piana, S.; Dror, R. O.; Eastwood, M. P.; Bank, J. A.; Jumper, J. M.; Salmon, J. K.; Shan, Y.et al. Atomic-Level Characterization of the Structural Dynamics of Proteins. Science 2010, 330, 341-346.

(37) Shelley, J. C.; Shelley, M. Y.; Reeder, R. C.; Bandyopadhyay, S.; Klein, M. L. A Coarse Grain Model for Phospholipid Simulations. The Journal of Physical Chemistry B 2001, 105, 44644470.

(38) Shelley, J. C.; Shelley, M. Y.; Reeder, R. C.; Bandyopadhyay, S.; Moore, P. B.; Klein, M. L. Simulations of Phospholipids Using a Coarse Grain Model. The Journal of Physical Chemistry B 2001, 105, 9785-9792.

(39) Marrink, S. J.; de Vries, A. H.; Mark, A. E. Coarse Grained Model for Semiquantitative Lipid Simulations. The Journal of Physical Chemistry B 2004, 108, 750-760.

(40) Lopez, C. A.; Sovova, Z.; van Eerden, F. J.; de Vries, A. H.; Marrink, S. J. Martini Force Field Parameters for Glycolipids. J Chem Theory Comput 2013, 9, 1694-1708.

(41) Saunders, M. G.; Voth, G. A. Coarse-Graining Methods for Computational Biology. Annu Rev Biophys 2013, 42, 73-93.

(42) Zwanzig, R. W. High-Temperature Equation of State by a Perturbation Method. I. Nonpolar Gases. J. Chem. Phys. 1954, 22.

(43) Beveridge, D. L.; DiCapua, F. M. Free Energy Via Molecular Simulation: Applications to Chemical and Biomolecular Systems. Annual Review of Biophysics and Biophysical Chemistry 1989, 18, 431-492.

(44) Wang, L.; Wu, Y. J.; Deng, Y. Q.; Kim, B.; Pierce, L.; Krilov, G.; Lupyan, D.; Robinson, S.; Dahlgren, M. K.; Greenwood, J.et al. Accurate and Reliable Prediction of Relative Ligand Binding Potency in Prospective Drug Discovery by Way of a Modern Free-Energy Calculation Protocol and Force Field. Journal of the American Chemical Society 2015, 137, 2695-2703.

(45) Torrie, G. M.; Valleau, J. P. Monte Carlo Free Energy Estimates Using Non-Boltzmann Sampling: Application to the Sub-Critical Lennard-Jones Fluid. Chemical Physics Letters 1974, 28, 578-581. 
(46) Kumar, S.; Rosenberg, J. M.; Bouzida, D.; Swendsen, R. H.; Kollman, P. A. The Weighted Histogram Analysis Method for Free-Energy Calculations on Biomolecules. I. The Method. Journal of Computational Chemistry 1992, 13, 1011-1021.

(47) Souaille, M.; Roux, B. t. Extension to the Weighted Histogram Analysis Method: Combining Umbrella Sampling with Free Energy Calculations. Computer Physics Communications 2001, $135,40-57$.

(48) Kästner, J.; Thiel, W. Bridging the Gap between Thermodynamic Integration and Umbrella Sampling Provides a Novel Analysis Method: "Umbrella Integration". The Journal of Chemical Physics 2005, 123, 144104.

(49) Park, S.; Schulten, K. Calculating Potentials of Mean Force from Steered Molecular Dynamics Simulations. J Chem Phys 2004, 120, 5946-5961.

(50) Park, S.; Khalili-Araghi, F.; Tajkhorshid, E.; Schulten, K. Free Energy Calculation from Steered Molecular Dynamics Simulations Using Jarzynski's Equality. The Journal of Chemical Physics 2003, 119, 3559-3566.

(51) Furini, S.; Domene, C. Computational Studies of Transport in Ion Channels Using Metadynamics. Biochimica et Biophysica Acta (BBA) - Biomembranes 2016, 1858, 1733 1740 .

(52) Darve, E.; Pohorille, A. Calculating Free Energies Using Average Force. The Journal of Chemical Physics 2001, 115, 9169-9183.

(53) Meng, X. Y.; Zhang, H. X.; Mezei, M.; Cui, M. Molecular Docking: A Powerful Approach for Structure-Based Drug Discovery. Current Computer-Aided Drug Design 2011, 7, 146-157.

(54) Pagadala, N. S.; Syed, K.; Tuszynski, J. Software for Molecular Docking: A Review. Biophysical Reviews 2017, 9, 91-102.

(55) Brannigan, G.; LeBard, D. N.; Henin, J.; Eckenhoff, R. G.; Klein, M. L. Multiple Binding Sites for the General Anesthetic Isoflurane Identified in the Nicotinic Acetylcholine Receptor Transmembrane Domain. Proceedings of the National Academy of Sciences of the United States of America 2010, 107, 14122-14127.

(56) Pickholz, M.; Giupponi, G. Coarse Grained Simulations of Local Anesthetics Encapsulated into a Liposome. Journal of Physical Chemistry B 2010, 114, 7009-7015.

(57) Pickholz, M.; Saiz, L.; Klein, M. L. Concentration Effects of Volatile Anesthetics on the Properties of Model Membranes: A Coarse-Grain Approach. Biophysical Journal 2005, 88, 1524-1534.

(58) Huang, P.; Bertaccini, E.; Loew, G. H. Molecular-Dynamics Simulation of AnestheticPhospholipid Bilayer Interactions. Journal of Biomolecular Structure \& Dynamics 1995, 12, 725-754.

(59) Pohorille, A.; Cieplak, P.; Wilson, M. A. Interactions of Anesthetics with the MembraneWater Interface. Chemical Physics 1996, 204, 337-345.

(60) Ahumada, H.; Montecinos, R.; Tieleman, D. P.; Weiss-Lopez, B. E. Orientation and Dynamics of Benzyl Alcohol and Benzyl Alkyl Ethers Dissolved in Nematic Lyotropic Liquid Crystals. H$2 \mathrm{Nmr}$ and Molecular Dynamics Simulations. Journal of Physical Chemistry A 2005, 109, 6644-6651. 
(61) Castro, V.; Stevensson, B.; Dvinskikh, S. V.; Hogberg, C. J.; Lyubartsev, A. P.; Zimmermann, H.; Sandstrom, D.; Maliniak, A. Nmr Investigations of Interactions between Anesthetics and Lipid Bilayers. Biochimica Et Biophysica Acta-Biomembranes 2008, 1778, 2604-2611.

(62) Klacsova, M.; Bulacu, M.; Kucerka, N.; Uhrikova, D.; Teixeira, J.; Marrink, S. J.; Balgavy, P. The Effect of Aliphatic Alcohols on Fluid Bilayers in Unilamellar Dopc Vesicles - a SmallAngle Neutron Scattering and Molecular Dynamics Study. Biochimica Et Biophysica ActaBiomembranes 2011, 1808, 2136-2146.

(63) McCarthy, N. L. C.; Brooks, N. J.; Tyler, A. I. I.; ElGamacy, M.; Welche, P. R. L.; Payne, M. C.; Chau, P. L. A Combined X-Ray Scattering and Simulation Study of Halothane in Membranes at Raised Pressures. Chemical Physics Letters 2017, 671, 21-27.

(64) Porasso, R. D.; Bennett, W. F. D.; Oliveira-Costa, S. D.; Cascales, J. J. L. Study of the Benzocaine Transfer from Aqueous Solution to the Interior of a Biological Membrane. Journal of Physical Chemistry B 2009, 113, 9988-9994.

(65) Cascales, J. J. L.; Costa, S. D. O.; Porasso, R. D. Thermodynamic Study of Benzocaine Insertion into Different Lipid Bilayers. Journal of Chemical Physics 2011, 135, 7.

(66) Cascales, J. J. L.; Costa, S. D. O. Effect of the Interfacial Tension and Ionic Strength on the Thermodynamic Barrier Associated to the Benzocaine Insertion into a Cell Membrane. Biophysical Chemistry 2013, 172, 1-7.

(67) Polley, A.; Vemparala, S. Partitioning of Ethanol in Multi-Component Membranes: Effects on Membrane Structure. Chemistry and Physics of Lipids 2013, 166, 1-11.

(68) Reigada, R. Atomistic Study of Lipid Membranes Containing Chloroform: Looking for a Lipid-Mediated Mechanism of Anesthesia. Plos One 2013, 8, 10.

(69) Booker, R. D.; Sum, A. K. Biophysical Changes Induced by Xenon on Phospholipid Bilayers. Biochimica Et Biophysica Acta-Biomembranes 2013, 1828, 1347-1356.

(70) Guixa-Gonzalez, R.; Rodriguez-Espigares, I.; Ramirez-Anguita, J. M.; Carrio-Gaspar, P.; Martinez-Seara, H.; Giorgino, T.; Selent, J. Membplugin: Studying Membrane Complexity in Vmd. Bioinformatics 2014, 30, 1478-1480.

(71) Mojumdar, E. H.; Lyubartsev, A. P. Molecular Dynamics Simulations of Local Anesthetic Articaine in a Lipid Bilayer. Biophysical Chemistry 2010, 153, 27-35.

(72) Amjad-Iranagh, S.; Yousefpour, A.; Haghighi, P.; Modarress, H. Effects of Protein Binding on a Lipid Bilayer Containing Local Anesthetic Articaine, and the Potential of Mean Force Calculation: A Molecular Dynamics Simulation Approach. Journal of Molecular Modeling 2013, 19, 3831-3842.

(73) Saeedi, M.; Lyubartsev, A. P.; Jalili, S. Anesthetics Mechanism on a Dmpc Lipid Membrane Model: Insights from Molecular Dynamics Simulations. Biophysical Chemistry 2017, 226, 113.

(74) Skjevik, A. A.; Haug, B. E.; Lygre, H.; Teigen, K. Intramolecular Hydrogen Bonding in Articaine Can Be Related to Superior Bone Tissue Penetration: A Molecular Dynamics Study. Biophysical Chemistry 2011, 154, 18-25.

(75) Bernardi, R. C.; Pascutti, P. G. Hybrid Qm/Mm Molecular Dynamics Study of Benzocaine in a Membrane Environment: How Does a Quantum Mechanical Treatment of Both Anesthetic and Lipids Affect Their Interaction. Journal of Chemical Theory and Computation 2012, 8, 2197-2203. 
(76) Martin, L. J.; Chao, R.; Corry, B. Molecular Dynamics Simulation of the Partitioning of Benzocaine and Phenytoin into a Lipid Bilayer. Biophysical Chemistry 2014, 185, 98-107.

(77) Cascales, J. J. L.; Cifre, J. G. H.; de la Torre, J. G. Anaesthetic Mechanism on a Model Biological Membrane: A Molecular Dynamics Simulation Study. Journal of Physical Chemistry B 1998, 102, 625-631.

(78) Martini, M. F.; Pickholz, M. Molecular Dynamics Study of Uncharged Bupivacaine Enantiomers in Phospholipid Bilayers. International Journal of Quantum Chemistry 2012, 112, 3341-3345.

(79) Pickholz, M.; Fraceto, L. F.; de Paula, E. Preferential Location of Prilocaine and Etidocaine in Phospholipid Bilayers: A Molecular Dynamics Study. Synthetic Metals 2009, 159, 21572158.

(80) Pasenkiewicz-Gierula, M.; Rog, T.; Grochowski, J.; Serda, P.; Czarnecki, R.; Librowski, T.; Lochynski, S. Effects of a Carane Derivative Local Anesthetic on a Phospholipid Bilayer Studied by Molecular Dynamics Simulation. Biophysical Journal 2003, 85, 1248-1258.

(81) Hogberg, C. J.; Maliniak, A.; Lyubartsev, A. P. Dynamical and Structural Properties of Charged and Uncharged Lidocaine in a Lipid Bilayer. Biophysical Chemistry 2007, 125, 416 424.

(82) Hogberg, C. J.; Lyubartsev, A. P. Effect of Local Anesthetic Lidocaine on Electrostatic Properties of a Lipid Bilayer. Biophysical Journal 2008, 94, 525-531.

(83) Zapata-Morin, P. A.; Sierra-Valdez, F. J.; Ruiz-Suarez, J. C. The Interaction of Local Anesthetics with Lipid Membranes. Journal of Molecular Graphics \& Modelling 2014, 53, 200-205.

(84) Pickholz, M.; Fraceto, L. F.; de Paula, E. Distribution of Neutral Prilocaine in a Phospholipid Bilayer: Insights from Molecular Dynamics Simulations. International Journal of Quantum Chemistry 2008, 108, 2386-2391.

(85) Jalili, S.; Saeedi, M. Study of Procaine and Tetracaine in the Lipid Bilayer Using Molecular Dynamics Simulation. European Biophysics Journal with Biophysics Letters 2017, 46, 265282.

(86) Kondela, T.; Gallova, J.; Hauss, T.; Barnoud, J.; Marrink, S. J.; Kucerka, N. Alcohol Interactions with Lipid Bilayers. Molecules 2017, 22, 15.

(87) Griepernau, B.; Leis, S.; Schneider, M. F.; Sikor, M.; Steppich, D.; Bockmann, R. A. 1Alkanols and Membranes: A Story of Attraction. Biochimica Et Biophysica ActaBiomembranes 2007, 1768, 2899-2913.

(88) Griepernau, B.; Bockmann, R. A. The Influence of 1-Alkanols and External Pressure on the Lateral Pressure Profiles of Lipid Bilayers. Biophysical Journal 2008, 95, 5766-5778.

(89) Terama, E.; Ollila, O. H. S.; Salonen, E.; Rowat, A. C.; Trandum, C.; Westh, P.; Patra, M.; Karttunen, M.; Vattulainen, I. Influence of Ethanol on Lipid Membranes: From Lateral Pressure Profiles to Dynamics and Partitioning. Journal of Physical Chemistry B 2008, 112, 4131-4139.

(90) Reigada, R. Influence of Chloroform in Liquid-Ordered and Liquid-Disordered Phases in Lipid Membranes. Journal of Physical Chemistry B 2011, 115, 2527-2535. 
(91) Darvas, M.; Hoang, P. N. M.; Picaud, S.; Sega, M.; Jedlovszky, P. Anesthetic Molecules Embedded in a Lipid Membrane: A Computer Simulation Study. Physical Chemistry Chemical Physics 2012, 14, 12956-12969.

(92) Vorobyov, I.; Bennett, W. F. D.; Tieleman, D. P.; Allen, T. W.; Noskov, S. The Role of Atomic Polarization in the Thermodynamics of Chloroform Partitioning to Lipid Bilayers. Journal of Chemical Theory and Computation 2012, 8, 618-628.

(93) Fabian, B.; Darvas, M.; Picaud, S.; Sega, M.; Jedlovszky, P. The Effect of Anaesthetics on the Properties of a Lipid Membrane in the Biologically Relevant Phase: A Computer Simulation Study. Physical Chemistry Chemical Physics 2015, 17, 14750-14760.

(94) Fabian, B.; Sega, M.; Voloshin, V. P.; Medvedev, N. N.; Jedlovszky, P. Lateral Pressure Profile and Free Volume Properties in Phospholipid Membranes Containing Anesthetics. Journal of Physical Chemistry B 2017, 121, 2814-2824.

(95) Vemparala, S.; Saiz, L.; Eckenhoff, R. G.; Klein, M. L. Partitioning of Anesthetics into a Lipid Bilayer and Their Interaction with Membrane-Bound Peptide Bundles. Biophysical Journal 2006, 91, 2815-2825.

(96) Chau, P. L.; Jedlovszky, P.; Hoang, P. N. M.; Picaud, S. Pressure Reversal of General Anaesthetics: A Possible Mechanism from Molecular Dynamics Simulations. Journal of Molecular Liquids 2009, 147, 128-134.

(97) Chau, P. L.; Tu, K. M.; Liang, K. K.; Todorov, I. T.; Roser, S. J.; Barker, R.; Matubayasi, N. The Effect of Pressure on Halothane Binding to Hydrated Dmpc Bilayers. Molecular Physics 2012, 110, 1461-1467.

(98) Chau, P. L.; Hoang, P. N. M.; Picaud, S.; Jedlovszky, P. A Possible Mechanism for Pressure Reversal of General Anaesthetics from Molecular Simulations. Chemical Physics Letters 2007, 438, 294-297.

(99) Tu, K. C.; Tarek, M.; Klein, M. L.; Scharf, D. Effects of Anesthetics on the Structure of a Phospholipid Bilayer: Molecular Dynamics Investigation of Halothane in the Hydrated Liquid Crystal Phase of Dipalmitoylphosphatidylcholine. Biophysical Journal 1998, 75, 2123 2134.

(100) Koubi, L.; Tarek, M.; Klein, M. L.; Scharf, D. Distribution of Halothane in a Dipalmitoyl Phosphatidylcholine Bilayer from Molecular Dynamics Calculations. Biophysical Journal 2000, 78, 800-811.

(101) Tu, K. M.; Matubayasi, N.; Liang, K. K.; Todorov, I. T.; Chan, S. L.; Chau, P. L. A Possible Molecular Mechanism for the Pressure Reversal of General Anaesthetics: Aggregation of Halothane in Popc Bilayers at High Pressure. Chemical Physics Letters 2012, 543, 148-154.

(102) Koubi, L.; Saiz, L.; Tarek, M.; Scharf, D.; Klein, M. L. Influence of Anesthetic and Nonimmobilizer Molecules on the Physical Properties of a Polyunsaturated Lipid Bilayer. Journal of Physical Chemistry B 2003, 107, 14500-14508.

(103) Arcario, M. J.; Mayne, C. G.; Tajkhorshid, E. Atomistic Models of General Anesthetics for Use in in Silico Biological Studies. Journal of Physical Chemistry B 2014, 118, 12075-12086.

(104) Wieteska, J. R.; Welche, P. R. L.; Tu, K. M.; ElGamacy, M.; Csanyi, G.; Payne, M. C.; Chaue, P. L. Isoflurane Does Not Aggregate inside Popc Bilayers at High Pressure: Implications for Pressure Reversal of General Anaesthesia. Chemical Physics Letters 2015, 638, 116-121. 
(105) Jerabek, H.; Pabst, G.; Rappolt, M.; Stockner, T. Membrane-Mediated Effect on Ion Channels Induced by the Anesthetic Drug Ketamine. Journal of the American Chemical Society 2010, 132, 7990-7997.

(106) Hansen, A. H.; Sorensen, K. T.; Mathieu, R.; Serer, A.; Duelund, L.; Khandelia, H.; Hansen, P. L.; Simonsen, A. C. Propofol Modulates the Lipid Phase Transition and Localizes near the Headgroup of Membranes. Chemistry and Physics of Lipids 2013, 175, 84-91.

(107) Stimson, L. M.; Vattulainen, I.; Rog, T.; Karttunen, M. Exploring the Effect of Xenon on Biomembranes. Cellular \& Molecular Biology Letters 2005, 10, 563-569.

(108) Chen, J. L.; Chen, L.; Wang, Y.; Wang, X. G.; Zeng, S. W. Exploring the Effects on Lipid Bilayer Induced by Noble Gases Via Molecular Dynamics Simulations. Scientific Reports 2015, 5, 6.

(109) Yamamoto, E.; Akimoto, T.; Shimizu, H.; Hirano, Y.; Yasui, M.; Yasuoka, K. Diffusive Nature of Xenon Anesthetic Changes Properties of a Lipid Bilayer: Molecular Dynamics Simulations. Journal of Physical Chemistry B 2012, 116, 8989-8995.

(110) Koubi, L.; Tarek, M.; Bandyopadhyay, S.; Klein, M. L.; Scharf, D. Membrane Structural Perturbations Caused by Anesthetics and Nonimmobilizers: A Molecular Dynamics Investigation. Biophysical Journal 2001, 81, 3339-3345.

(111) Koubi, L.; Tarek, M.; Bandyopadhyay, S.; Klein, M. L.; Scharf, D. Effects of the Nonimmobilizer Hexafluroethane on the Model Membrane Dimyristoylphosphatidylcholine. Anesthesiology 2002, 97, 848-855.

(112) Kandel, L.; Chortkoff, B. S.; Sonner, J.; Laster, M. J.; Eger, E. I. Nonanesthetics Can Suppress Learning. Anesthesia and Analgesia 1996, 82, 321-326.

(113) Baber, J.; Ellena, J. F.; Cafiso, D. S. Distribution of General-Anesthetics in PhospholipidBilayers Determined Using H-2 Nmr and H-1-H-1 Noe Spectroscopy. Biochemistry 1995, 34, 6533-6539.

(114) North, C.; Cafiso, D. S. Contrasting Membrane Localization and Behavior of Halogenated Cyclobutanes That Follow or Violate the Meyer-Overton Hypothesis of General Anesthetic Potency. Biophysical Journal 1997, 72, 1754-1761.

(115) Pohorille, A.; Wilson, M. A.; New, M. H.; Chipot, C. Concentrations of Anesthetics across the Water-Membrane Interface; the Meyer-Overton Hypothesis Revisited. Toxicology Letters 1998, 101, 421-430.

(116) Pohorille, A.; Wilson, M. A. Excess Chemical Potential of Small Solutes across WaterMembrane and Water-Hexane Interfaces. Journal of Chemical Physics 1996, 104, 37603773.

(117) Strichartz, G. Molecular Mechanisms of Nerve Block by Local Anesthetics. Anesthesiology 1976, 45, 421-441.

(118) Pasenkiewicz-Gierula, M.; Rog, T.; Grochowski, J.; Serda, P.; Czarnecki, R.; Librowski, T.; Lochynski, S. Effects of a Carane Derivative Local Anesthetic on a Phospholipid Bilayer Studied by Molecular Dynamics Simulation. Biophys J 2003, 85, 1248-1258.

(119) Hata, T.; Sakamoto, T.; Matsuki, H.; Kaneshina, S. Partition Coefficients of Charged and Uncharged Local Anesthetics into Dipalmitoylphosphatidylcholine Bilayer Membrane: Estimation from Ph Dependence on the Depression of Phase Transition Temperatures. Colloids Surf B Biointerfaces 2001, 22, 77-84. 
(120) Johnson, F. H.; Flagler, E. A. Hydrostatic Pressure Reversal of Narcosis in Tadpoles. Science 1950, 112, 91-92.

(121) Johnson, F. H.; Flagler, E. A. Activity of Narcotized Amphibian Larvae under Hydrostatic Pressure. Journal of Cellular and Comparative Physiology 1951, 37, 15-25.

(122) Sanders, R. D.; Franks, N. P.; Maze, M. Xenon: No Stranger to Anaesthesia. British Journal of Anaesthesia 2003, 91, 709-717.

(123) Harris, P. D.; Barnes, R. The Uses of Helium and Xenon in Current Clinical Practice. Anaesthesia 2008, 63, 284-293.

(124) Brucken, A.; Coburn, M.; Rex, S.; Rossaint, R.; Fries, M. Current Developments in Xenon Research. Anaesthesist 2010, 59, 883-+.

(125) Cullen, S. C.; Gross, E. G. The Anesthetic Properties of Xenon in Animals and Human Beings, with Additional Observations on Krypton. Science 1951, 113, 580-582.

(126) Franks, N. P.; Dickinson, R.; de Sousa, S. L. M.; Hall, A. C.; Lieb, W. R. How Does Xenon Produce Anaesthesia? Nature 1998, 396, 324-324.

(127) de Sousa, S. L. M.; Dickinson, R.; Lieb, W. R.; Franks, N. P. Contrasting Synaptic Actions of the Inhalational General Anesthetics Isoflurane and Xenon. Anesthesiology 2000, 92, 10551066.

(128) Yamakura, T.; Harris, R. A. Effects of Gaseous Anesthetics Nitrous Oxide and Xenon on Ligand-Gated Ion Channels: Comparison with Isoflurane and Ethanol. Anesthesiology 2000, 93, 1095-1101.

(129) Gruss, M.; Bushell, T. J.; Bright, D. P.; Lieb, W. R.; Mathie, A.; Franks, N. P. Two-PoreDomain K+ Channels Are a Novel Target for the Anesthetic Gases Xenon, Nitrous Oxide, and Cyclopropane. Molecular Pharmacology 2004, 65, 443-452.

(130) Cantor, R. S. Breaking the Meyer-Overton Rule: Pedicted Effects of Varying Stiffness and Interfacial Activity on the Intrinsic Potency of Anesthetics. Biophysical Journal 2001, 80, 2284-2297.

(131) Corringer, P. J.; Poitevin, F.; Prevost, M. S.; Sauguet, L.; Delarue, M.; Changeux, J. P. Structure and Pharmacology of Pentameric Receptor Channels: From Bacteria to Brain. Structure 2012, 20, 941-956.

(132) Miller, K. W. The Nature of Sites of General Anaesthetic Action. British Journal of Anaesthesia 2002, 89, 17-31.

(133) Yamakura, T.; Bertaccini, E.; Trudell, J. R.; Harris, R. A. Anesthetics and Ion Channels: Molecular Models and Sites of Action. Annual Review of Pharmacology and Toxicology 2001, 41, 23-51.

(134) Unwin, N. Refined Structure of the Nicotinic Acetylcholine Receptor at 4 Angstrom Resolution. Journal of Molecular Biology 2005, 346, 967-989.

(135) Thompson, A. J.; Lester, H. A.; Lummis, S. C. R. The Structural Basis of Function in Cys-Loop Receptors. Quarterly Reviews of Biophysics 2010, 43, 449-499.

(136) Hilf, R. J. C.; Dutzler, R. X-Ray Structure of a Prokaryotic Pentameric Ligand-Gated Ion Channel. Nature 2008, 452, 375-U312.

(137) Pan, J. J.; Chen, Q.; Willenbring, D.; Yoshida, K.; Tillman, T.; Kashlan, O. B.; Cohen, A.; Kong, X. P.; Xu, Y.; Tang, P. Structure of the Pentameric Ligand-Gated Ion Channel Elic 
Cocrystallized with Its Competitive Antagonist Acetylcholine. Nature Communications 2012, 3, 8.

(138) Bocquet, N.; Nury, H.; Baaden, M.; Le Poupon, C.; Changeux, J. P.; Delarue, M.; Corringer, P. J. X-Ray Structure of a Pentameric Ligand-Gated Ion Channel in an Apparently Open Conformation. Nature 2009, 457, 111-114.

(139) Hilf, R. J. C.; Dutzler, R. Structure of a Potentially Open State of a Proton-Activated Pentameric Ligand-Gated Ion Channel. Nature 2009, 457, 115-U122.

(140) Weng, Y.; Yang, L. Y.; Corringer, P. J.; Sonner, J. M. Anesthetic Sensitivity of the Gloeobacter Violaceus Proton-Gated Ion Channel. Anesthesia and Analgesia 2010, 110, 59-63.

(141) Cheng, M. H.; Coalson, R. D.; Cascio, M. Molecular Dynamics Simulations of Ethanol Binding to the Transmembrane Domain of the Glycine Receptor: Implications for the Channel Potentiation Mechanism. Proteins-Structure Function and Bioinformatics 2008, 71, $972-$ 981.

(142) Cheng, M. H. Y.; Cascio, M.; Coalson, R. D. Homology Modeling and Molecular Dynamics Simulations of the Alpha 1 Glycine Receptor Reveals Different States of the Channel. Proteins-Structure Function and Bioinformatics 2007, 68, 581-593.

(143) Murail, S.; Wallner, B.; Trudell, J. R.; Bertaccini, E.; Lindahl, E. Microsecond Simulations Indicate That Ethanol Binds between Subunits and Could Stabilize an Open-State Model of a Glycine Receptor. Biophysical Journal 2011, 100, 1642-1650.

(144) Haddadian, E. J.; Cheng, M. H. Y.; Coalson, R. D.; Xu, Y.; Tang, P. In Silico Models for the Human Alpha 4 Beta 2 Nicotinic Acetylcholine Receptor. Journal of Physical Chemistry $B$ 2008, 112, 13981-13990.

(145) Liu, L. T.; Haddadian, E. J.; Willenbring, D.; Xu, Y.; Tang, P. Higher Susceptibility to Halothane Modulation in Open- Than in Closed-Channel Alpha 4 Beta 2 Nachr Revealed by Molecular Dynamics Simulations. Journal of Physical Chemistry B 2010, 114, 626-632.

(146) Liu, L. T.; Willenbring, D.; Xu, Y.; Tang, P. General Anesthetic Binding to Neuronal Alpha 4 Beta 2 Nicotinic Acetylcholine Receptor and Its Effects on Global Dynamics. Journal of Physical Chemistry B 2009, 113, 12581-12589.

(147) Yoluk, O.; Bromstrup, T.; Bertaccini, E. J.; Trudell, J. R.; Lindahl, E. Stabilization of the Glucl Ligand-Gated Ion Channel in the Presence and Absence of Ivermectin. Biophysical Journal 2013, 105, 640-647.

(148) Carpenter, T. S.; Lau, E. Y.; Lightstone, F. C. A Role for Loop F in Modulating Gaba Binding Affinity in the Gaba(a) Receptor. Journal of Molecular Biology 2012, 422, 310-323.

(149) Carpenter, T. S.; Lau, E. Y.; Lightstone, F. C. Identification of a Possible Secondary Picrotoxin-Binding Site on the Gaba(a) Receptor. Chemical Research in Toxicology 2013, 26, 1444-1454.

(150) Henin, J.; Salari, R.; Murlidaran, S.; Brannigan, G. A Predicted Binding Site for Cholesterol on the Gaba(a) Receptor. Biophysical Journal 2014, 106, 1938-1949.

(151) Woll, K. A.; Murlidaran, S.; Pinch, B. J.; Henin, J.; Wang, X. S.; Salari, R.; Covarrubias, M.; Dailey, W. P.; Brannigan, G.; Garcia, B. A.et al. A Novel Bifunctional Alkylphenol Anesthetic Allows Characterization of Gamma-Aminobutyric Acid, Type a (Gaba(a)), Receptor Subunit Binding Selectivity in Synaptosomes. Journal of Biological Chemistry 2016, 291, 2047320486. 
(152) Chiara, D. C.; Dangott, L. J.; Eckenhoff, R. G.; Cohen, J. B. Identification of Nicotinic Acetylcholine Receptor Amino Acids Photolabeled by the Volatile Anesthetic Halothane. Biochemistry 2003, 42, 13457-13467.

(153) Nury, H.; Van Renterghem, C.; Weng, Y.; Tran, A.; Baaden, M.; Dufresne, V.; Changeux, J. P.; Sonner, J. M.; Delarue, M.; Corringer, P. J. X-Ray Structures of General Anaesthetics Bound to a Pentameric Ligand-Gated Ion Channel. Nature 2011, 469, 428-+.

(154) Fourati, Z.; Howard, R. J.; Heusser, S. A.; Hu, H. D.; Ruza, R. R.; Sauguet, L.; Lindahl, E.; Delarue, M. Structural Basis for a Bimodal Allosteric Mechanism of General Anesthetic Modulation in Pentameric Ligand-Gated Ion Channels. Cell Reports 2018, 23, 993-1004.

(155) Sauguet, L.; Howard, R. J.; Malherbe, L.; Lee, U. S.; Corringer, P. J.; Harris, R. A.; Delarue, M. Structural Basis for Potentiation by Alcohols and Anaesthetics in a Ligand-Gated Ion Channel. Nature Communications 2013, 4, 10.

(156) Laurent, B.; Murail, S.; Shahsavar, A.; Sauguet, L.; Delarue, M.; Baaden, M. Sites of Anesthetic Inhibitory Action on a Cationic Ligand-Gated Ion Channel. Structure 2016, 24, 595-605.

(157) Chen, Q.; Kinde, M. N.; Arjunan, P.; Wells, M. M.; Cohen, A. E.; Xu, Y.; Tang, P. Direct Pore Binding as a Mechanism for Isoflurane Inhibition of the Pentameric Ligand-Gated Ion Channel Elic. Scientific Reports 2015, 5, 11.

(158) Pan, J. J.; Chen, Q.; Willenbring, D.; Mowrey, D.; Kong, X. P.; Cohen, A.; Divito, C. B.; Xu, Y.; Tang, P. Structure of the Pentameric Ligand-Gated Ion Channel Glic Bound with Anesthetic Ketamine. Structure 2012, 20, 1463-1469.

(159) Willenbring, D.; Liu, L. T.; Mowrey, D.; Xu, Y.; Tang, P. Isoflurane Alters the Structure and Dynamics of Glic. Biophysical Journal 2011, 101, 1905-1912.

(160) Arcario, M. J.; Mayne, C. G.; Tajkhorshid, E. A Membrane-Embedded Pathway Delivers General Anesthetics to Two Interacting Binding Sites in the Gloeobacter Violaceus Ion Channel. Journal of Biological Chemistry 2017, 292, 9480-9492.

(161) Chen, Q. A.; Cheng, M. H.; Xu, Y.; Tang, P. Anesthetic Binding in a Pentameric Ligand-Gated Ion Channel: Glic. Biophysical Journal 2010, 99, 1801-1809.

(162) Kash, T. L.; Jenkins, A.; Kelley, J. C.; Trudell, J. R.; Harrison, N. L. Coupling of Agonist Binding to Channel Gating in the Gaba(a) Receptor. Nature 2003, 421, 272-275.

(163) Xiu, X. N.; Hanek, A. P.; Wang, J. T.; Lester, H. A.; Dougherty, D. A. A Unified View of the Role of Electrostatic Interactions in Modulating the Gating of Cys Loop Receptors. Journal of Biological Chemistry 2005, 280, 41655-41666.

(164) Jha, A.; Cadugan, D. J.; Purohit, P.; Auerbach, A. Acetylcholine Receptor Gating at Extracellular Transmembrane Domain Interface: The Cys-Loop and M2-M3 Linker. Journal of General Physiology 2007, 130, 547-558.

(165) Cheng, M. H. Y.; Coalson, R. D.; Tang, P. Molecular Dynamics and Brownian Dynamics Investigation of Ion Permeation and Anesthetic Halothane Effects on a Proton-Gated Ion Channel. Journal of the American Chemical Society 2010, 132, 16442-16449.

(166) Lee, W. Y.; Sine, S. M. Principal Pathway Coupling Agonist Binding to Channel Gating in Nicotinic Receptors. Nature 2005, 438, 243-247. 
(167) Sala, F.; Mulet, J.; Sala, S.; Gerber, S.; Criado, M. Charged Amino Acids of the N-Terminal Domain Are Involved in Coupling Binding and Gating in Alpha 7 Nicotinic Receptors. Journal of Biological Chemistry 2005, 280, 6642-6647.

(168) Joseph, T. T.; Mincer, J. S. Common Internal Allosteric Network Links Anesthetic Binding Sites in a Pentameric Ligand-Gated Ion Channel. Plos One 2016, 11, 20.

(169) Mowrey, D.; Cheng, M. H.; Liu, L. T.; Willenbring, D.; Lu, X. H.; Wymore, T.; Xu, Y.; Tang, P. Asymmetric Ligand Binding Facilitates Conformational Transitions in Pentameric LigandGated Ion Channels. Journal of the American Chemical Society 2013, 135, 2172-2180.

(170) Rayes, D.; De Rosa, M. J.; Sine, S. M.; Bouzat, C. Number and Locations of Agonist Binding Sites Required to Activate Homomeric Cys-Loop Receptors. Journal of Neuroscience 2009, 29, 6022-6032.

(171) Beato, M.; Groot-Kormelink, P. J.; Colquhoun, D.; Sivilotti, L. G. The Activation Mechanism of Alpha 1 Homomeric Glycine Receptors. Journal of Neuroscience 2004, 24, 895-906.

(172) Amin, J.; Weiss, D. S. Insights into the Activation Mechanism of P(1) Gaba Receptors Obtained by Coexpression of Wild Type and Activation-Impaired Subunits. Proceedings of the Royal Society B-Biological Sciences 1996, 263, 273-282.

(173) Hilf, R. J. C.; Bertozzi, C.; Zimmermann, I.; Reiter, A.; Trauner, D.; Dutzler, R. Structural Basis of Open Channel Block in a Prokaryotic Pentameric Ligand-Gated Ion Channel. Nature Structural \& Molecular Biology 2010, 17, 1330-U1184.

(174) LeBard, D. N.; Henin, J.; Eckenhoff, R. G.; Klein, M. L.; Brannigan, G. General Anesthetics Predicted to Block the Glic Pore with Micromolar Affinity. Plos Computational Biology 2012, 8, 9.

(175) Nirthanan, S.; Garcia, G.; Chiara, D. C.; Husain, S. S.; Cohen, J. B. Identification of Binding Sites in the Nicotinic Acetylcholine Receptor for Tdbzl-Etomidate, a Photoreactive Positive Allosteric Effector. Journal of Biological Chemistry 2008, 283, 22051-22062.

(176) Ion, B. F.; Wells, M. M.; Chen, Q.; Xu, Y.; Tang, P. Ketamine Inhibition of the Pentameric Ligand-Gated Ion Channel Glic. Biophysical Journal 2017, 113, 605-612.

(177) Mowrey, D.; Chen, Q.; Liang, Y. H.; Liang, J.; Xu, Y.; Tang, P. Signal Transduction Pathways in the Pentameric Ligand-Gated Ion Channels. Plos One 2013, 8, 8.

(178) Li, X. Y.; Xie, F.; Zhang, J. C.; Su, J. G. Study, by Use of Coarse-Grained Models, of the Functionally Crucial Residues and Allosteric Pathway of Anesthetic Regulation of the Gloeobacter Violaceus Ligand-Gated Ion Channel. European Biophysics Journal with Biophysics Letters 2014, 43, 623-630.

(179) John, N. W.; James, B. Voltage-Gated Sodium Channel Blockers; Target Validation and Therapeutic Potential. Current Topics in Medicinal Chemistry 2005, 5, 529-537.

(180) Bagal, S. K.; Chapman, M. L.; Marron, B. E.; Prime, R.; Storer, R. I.; Swain, N. A. Recent Progress in Sodium Channel Modulators for Pain. Bioorg Med Chem Lett 2014, 24, 36903699.

(181) Brau, M. E.; Vogel, W.; Hempelmann, G. Fundamental Properties of Local Anesthetics: HalfMaximal Blocking Concentrations for Tonic Block of Na+ and $\mathrm{K}+$ Channels in Peripheral Nerve. Anesthesia and Analgesia 1998, 87, 885-889.

(182) Komai, H.; McDowell, T. S. Local Anesthetic Inhibition of Voltage-Activated Potassium Currents in Rat Dorsal Root Ganglion Neurons. Anesthesiology 2001, 94, 1089-1095. 
(183) Doyle, D. A.; Morais Cabral, J.; Pfuetzner, R. A.; Kuo, A.; Gulbis, J. M.; Cohen, S. L.; Chait, B. T.; MacKinnon, R. The Structure of the Potassium Channel: Molecular Basis of $\mathrm{K}^{+}$ Conduction and Selectivity. Science 1998, 280, 69-77.

(184) Smart, O. S.; Goodfellow, J. M.; Wallace, B. A. The Pore Dimensions of Gramicidin A. Biophysical Journal 1993, 65, 2455-2460.

(185) Heginbotham, L.; Lu, Z.; Abramson, T.; MacKinnon, R. Mutations in the K+ Channel Signature Sequence. Biophysical Journal 1994, 66, 1061-1067.

(186) Parsegian, A. Energy of an Ion Crossing a Low Dielectric Membrane: Solutions to Four Relevant Electrostatic Problems. Nature 1969, 221, 844-846.

(187) Jiang, Y. X.; Lee, A.; Chen, J. Y.; Ruta, V.; Cadene, M.; Chait, B. T.; MacKinnon, R. X-Ray Structure of a Voltage-Dependent K+ Channel. Nature 2003, 423, 33-41.

(188) Butterwick, J. A.; MacKinnon, R. Solution Structure and Phospholipid Interactions of the Isolated Voltage-Sensor Domain from Kvap. Journal of Molecular Biology 2010, 403, 591606.

(189) Long, S. B.; Campbell, E. B.; Mackinnon, R. Crystal Structure of a Mammalian VoltageDependent Shaker Family K+ Channel. Science 2005, 309, 897-903.

(190) Chen, X. R.; Wang, Q. H.; Ni, F. Y.; Ma, J. P. Structure of the Full-Length Shaker Potassium Channel Kv1.2 by Normal-Mode-Based X-Ray Crystallographic Refinement. Proceedings of the National Academy of Sciences of the United States of America 2010, 107, 11352-11357.

(191) Long, S. B.; Campbell, E. B.; Mackinnon, R. Voltage Sensor of Kv1.2: Structural Basis of Electromechanical Coupling. Science 2005, 309, 903-908.

(192) Long, S. B.; Tao, X.; Campbell, E. B.; MacKinnon, R. Atomic Structure of a VoltageDependent K+ Channel in a Lipid Membrane-Like Environment. Nature 2007, 450, 376-382.

(193) Payandeh, J.; Scheuer, T.; Zheng, N.; Catterall, W. A. The Crystal Structure of a VoltageGated Sodium Channel. Nature 2011, 475, 353-358.

(194) Payandeh, J.; Gamal El-Din, T. M.; Scheuer, T.; Zheng, N.; Catterall, W. A. Crystal Structure of a Voltage-Gated Sodium Channel in Two Potentially Inactivated States. Nature 2012, 486, 135-139.

(195) Lenaeus, M. J.; El-Din, T. M. G.; Ing, C.; Ramanadane, K.; Pomes, R.; Zheng, N.; Catterall, W. A. Structures of Closed and Open States of a Voltage-Gated Sodium Channel. Proceedings of the National Academy of Sciences of the United States of America 2017, 114, E3051E3060.

(196) Sula, A.; Booker, J.; Ng, L. C. T.; Naylor, C. E.; DeCaen, P. G.; Wallace, B. A. The Complete Structure of an Activated Open Sodium Channel. Nature Communications 2017, 8, 9.

(197) Zhang, X.; Ren, W.; DeCaen, P.; Yan, C.; Tao, X.; Tang, L.; Wang, J.; Hasegawa, K.; Kumasaka, T.; He, J.et al. Crystal Structure of an Orthologue of the Nachbac Voltage-Gated Sodium Channel. Nature 2012, 486, 130-134.

(198) Bagnéris, C.; DeCaen, P. G.; Hall, B. A.; Naylor, C. E.; Clapham, D. E.; Kay, C. W. M.; Wallace, B. A. Role of the C-Terminal Domain in the Structure and Function of Tetrameric Sodium Channels. Nat Commun 2013, 4. 
(199) McCusker, E. C.; Bagneris, C.; Naylor, C. E.; Cole, A. R.; D'Avanzo, N.; Nichols, C. G.; Wallace, B. A. Structure of a Bacterial Voltage-Gated Sodium Channel Pore Reveals Mechanisms of Opening and Closing. Nature Communications 2012, 3, 8.

(200) Bagneris, C.; DeCaen, P. G.; Naylor, C. E.; Pryde, D. C.; Nobeli, I.; Clapham, D. E.; Wallace, B. A. Prokaryotic Navms Channel as a Structural and Functional Model for Eukaryotic Sodium Channel Antagonism. Proceedings of the National Academy of Sciences of the United States of America 2014, 111, 8428-8433.

(201) Naylor, C. E.; Bagneris, C.; DeCaen, P. G.; Sula, A.; Scaglione, A.; Clapham, D. E.; Wallace, B. A. Molecular Basis of Ion Permeability in a Voltage-Gated Sodium Channel. Embo Journal 2016, 35, 820-830.

(202) Shaya, D.; Findeisen, F.; Abderemane-Ali, F.; Arrigoni, C.; Wong, S.; Nurva, S. R.; Loussouarn, G.; Minor Jr, D. L. Structure of a Prokaryotic Sodium Channel Pore Reveals Essential Gating Elements and an Outer Ion Binding Site Common to Eukaryotic Channels. Journal of Molecular Biology 2014, 426, 467-483.

(203) Shen, H. Z.; Zhou, Q.; Pan, X. J.; Li, Z. Q.; Wu, J. P.; Yan, N. Structure of a Eukaryotic VoltageGated Sodium Channel at near-Atomic Resolution. Science 2017, 355, 12.

(204) Wei, O. Y.; Hemmings, H. C. Isoform-Selective Effects of Isoflurane on Voltage-Gated Na+ Channels. Anesthesiology 2007, 107, 91-98.

(205) Shiraishi, M.; Harris, R. A. Effects of Alcohols and Anesthetics on Recombinant VoltageGated $\mathrm{Na}+$ Channels. Journal of Pharmacology and Experimental Therapeutics 2004, 309, 987-994.

(206) Clarkson, C. W.; Hondeghem, L. M. Mechanism for Bupivacaine Depression of Cardiac Conduction - Fast Block of Sodium-Channels During the Action-Potential with Slow Recovery from Block During Diastole. Anesthesiology 1985, 62, 396-405.

(207) Weidmann, S. The Effect of the Cardiac Membrane Potential on the Rapid Availability of the Sodium-Carrying System. Journal of Physiology-London 1955, 127, 213-224.

(208) Hille, B. Local-Anesthetics - Hydrophilic and Hydrophobic Pathways for Drug-Receptor Reaction. Journal of General Physiology 1977, 69, 497-515.

(209) Strichartz, G. R. The Inhibition of Sodium Currents in Myelinated Nerve by Quaternary Derivatives of Lidocaine. The Journal of General Physiology 1973, 62, 37-57.

(210) Khodorov, B.; Shishkova, L.; Peganov, E.; Revenko, S. Inhibition of Sodium Currents in Frog Ranvier Node Treated with Local-Anesthetics - Role of Slow Sodium Inactivation.

Biochimica Et Biophysica Acta 1976, 433, 409-435.

(211) Lee, S.; Goodchild, S. J.; Ahern, C. A. Local Anesthetic Inhibition of a Bacterial Sodium Channel. Journal of General Physiology 2012, 139, 507-516.

(212) Ouyang, W.; Jih, T. Y.; Zhang, T. T.; Correa, A. M.; Hemmings, H. C. Isoflurane Inhibits Nachbac, a Prokaryotic Voltage-Gated Sodium Channel. Journal of Pharmacology and Experimental Therapeutics 2007, 322, 1076-1083.

(213) Raju, S. G.; Barber, A. F.; LeBard, D. N.; Klein, M. L.; Carnevale, V. Exploring Volatile General Anesthetic Binding to a Closed Membrane-Bound Bacterial Voltage-Gated Sodium Channel Via Computation. Plos Computational Biology 2013, 9, 10.

(214) Barber, A. F.; Carnevale, V.; Klein, M. L.; Eckenhoff, R. G.; Covarrubias, M. Modulation of a Voltage-Gated $\mathrm{Na}+$ Channel by Sevoflurane Involves Multiple Sites and Distinct 
Mechanisms. Proceedings of the National Academy of Sciences of the United States of America 2014, 111, 6726-6731.

(215) Kinde, M. N.; Bondarenko, V.; Granata, D.; Bu, W. M.; Grasty, K. C.; Loll, P. J.; Carnevale, V.; Klein, M. L.; Eckenhoff, R. G.; Tang, P.et al. Fluorine-19 Nmr and Computational Quantification of Isoflurane Binding to the Voltage-Gated Sodium Channel Nachbac. Proceedings of the National Academy of Sciences of the United States of America 2016, 113, 13762-13767.

(216) Kaczmarski, J. A.; Corry, B. Investigating the Size and Dynamics of Voltage-Gated Sodium Channel Fenestrations a Molecular Dynamics Study. Channels 2014, 8, 14.

(217) Martin, L. J.; Corry, B. Locating the Route of Entry and Binding Sites of Benzocaine and Phenytoin in a Bacterial Voltage Gated Sodium Channel. Plos Computational Biology 2014, 10, 12.

(218) Ragsdale, D. S.; McPhee, J. C.; Scheuer, T.; Catterall, W. A. Molecular Determinants of State-Dependent Block of Na+ Channels by Local-Anesthetics. Science 1994, 265, 17241728.

(219) Ahern, C. A.; Eastwood, A. L.; Dougherty, D. A.; Horn, R. Electrostatic Contributions of Aromatic Residues in the Local Anesthetic Receptor of Voltage-Gated Sodium Channels. Circulation Research 2008, 102, 86-94.

(220) Smith, N. E.; Corry, B. Mutant Bacterial Sodium Channels as Models for Local Anesthetic Block of Eukaryotic Proteins. Channels 2016, 10, 225-237.

(221) Buyan, A.; Sun, D. L.; Corry, B. Protonation State of Inhibitors Determines Interaction Sites within Voltage-Gated Sodium Channels. Proceedings of the National Academy of Sciences of the United States of America 2018, 115, E3135-E3144.

(222) Patel, A. J.; Honore, E.; Lesage, F.; Fink, M.; Romey, G.; Lazdunski, M. Inhalational Anesthetics Activate Two-Pore-Domain Background K+ Channels. Nature Neuroscience 1999, 2, 422-426.

(223) Gibbons, S. J.; NunezHernandez, R.; Maze, G.; Harrison, N. L. Inhibition of a Fast Inwardly Rectifying Potassium Conductance by Barbiturates. Anesthesia and Analgesia 1996, 82, 1242-1246.

(224) Vemparala, S.; Domene, C.; Klein, M. L. Interaction of Anesthetics with Open and Closed Conformations of a Potassium Channel Studied Via Molecular Dynamics and Normal Mode Analysis. Biophysical Journal 2008, 94, 4260-4269.

(225) Domene, C.; Vemparala, S.; Klein, M. L.; Venien-Bryan, C.; Doyle, D. A. Role of Aromatic Localization in the Gating Process of a Potassium Channel. Biophysical Journal 2006, 90, L1L3.

(226) Tsao, D. H. H.; Casasfinet, J. R.; Maki, A. H.; Chase, J. W. Triplet-State Properties of Tryptophan Residues in Complexes of Mutated Escherichia-Coli Single-Stranded-DNA Binding-Proteins with Single-Stranded Polynucleotides. Biophysical Journal 1989, 55, 927 936.

(227) Davies, L. A.; Zhong, Q. F.; Klein, M. L.; Scharf, D. Molecular Dynamics Simulation of FourAlpha-Helix Bundles That Bind the Anesthetic Halothane. Febs Letters 2000, 478, 61-66.

(228) Tang, P.; Xu, Y. Large-Scale Molecular Dynamics Simulations of General Anesthetic Effects on the Ion Channel in the Fully Hydrated Membrane: The Implication of Molecular 
Mechanisms of General Anesthesia. Proceedings of the National Academy of Sciences of the United States of America 2002, 99, 16035-16040.

(229) Covarrubias, M.; Barber, A. F.; Carnevale, V.; Treptow, W.; Eckenhoff, R. G. Mechanistic Insights into the Modulation of Voltage-Gated Ion Channels by Inhalational Anesthetics. Biophysical Journal 2015, 109, 2003-2011.

(230) Correa, A. M. Gating Kinetics of Shaker K+ Channels Are Differentially Modified by General Anesthetics. American Journal of Physiology-Cell Physiology 1998, 275, C1009-C1021.

(231) Li, J. C.; Correa, A. M. Single-Channel Basis for Conductance Increase Induced by Isoflurane in Shaker H4ir K+ Channels. American Journal of Physiology-Cell Physiology 2001, 280, C1130-C1139.

(232) Tinklenberg, J. A.; Segal, I. S.; Tianzhi, G.; Maze, M. Analysis of Anesthetic Action on the Potassium Channels of the Shaker Mutant of Drosophila. Annals of the New York Academy of Sciences 1991, 625, 532-539.

(233) Li, J. C.; Correa, A. M. Kinetic Modulation of Herg Potassium Channels by the Volatile Anesthetic Halothane. Anesthesiology 2002, 97, 921-930.

(234) Friederich, P.; Benzenberg, D.; Trellakis, S.; Urban, B. W. Interaction of Volatile Anesthetics with Human Kv Channels in Relation to Clinical Concentrations. Anesthesiology 2001, 95, 954-958.

(235) Covarrubias, M.; Rubin, E. Ethanol Selectively Blocks a Noninactivating K+ Current Expressed in Xenopus-Oocytes. Proceedings of the National Academy of Sciences of the United States of America 1993, 90, 6957-6960.

(236) Covarrubias, M.; Vyas, T. B.; Escobar, L.; Wei, A. Alcohols Inhibit a Cloned Potassium Channel at a Discrete Saturable Site - Insights into the Molecular-Basis of GeneralAnesthesia. Journal of Biological Chemistry 1995, 270, 19408-19416.

(237) Harris, T.; Shahidullah, M.; Ellingson, J. S.; Covarrubias, M. General Anesthetic Action at an Internal Protein Site Involving the S4-S5 Cytoplasmic Loop of a Neuronal K+ Channel. Journal of Biological Chemistry 2000, 275, 4928-4936.

(238) Barber, A. F.; Liang, Q. S.; Covarrubias, M. Novel Activation of Voltage-Gated K+ Channels by Sevoflurane. Journal of Biological Chemistry 2012, 287, 40425-40432.

(239) Smith-Maxwell, C. J.; Ledwell, J. L.; Aldrich, R. W. Role of the S4 in Cooperativity of VoltageDependent Potassium Channel Activation. Journal of General Physiology 1998, 111, 399420.

(240) Ledwell, J. L.; Aldrich, R. W. Mutations in the S4 Region Isolate the Final Voltage-Dependent Cooperative Step in Potassium Channel Activation. Journal of General Physiology 1999, 113, 389-414.

(241) Chen, R.; Gryn'ova, G.; Wu, Y. L.; Coote, M. L.; Chung, S. H. Mechanisms and Energetics of Potassium Channel Block by Local Anesthetics and Antifungal Agents. Biochemistry 2014, $53,6786-6792$.

(242) Ander, M.; Luzhkov, V. B.; Aqvist, J. Ligand Binding to the Voltage-Gated Kv1.5 Potassium Channel in the Open State - Docking and Computer Simulations of a Homology Model. Biophysical Journal 2008, 94, 820-831.

(243) Decher, N.; Pirard, B.; Bundis, F.; Peukert, S.; Baringhaus, K. H.; Busch, A. E.; Steinmeyer, K.; Sanguinetti, M. C. Molecular Basis for Kv1.5 Channel Block - Conservation of Drug Binding 
Sites among Voltage-Gated K+ Channels. Journal of Biological Chemistry 2004, 279, $394-$ 400.

(244) Bradding, P.; Wulff, H. The K+ Channels K(Ca)3.1 and K(V)1.3 as Novel Targets for Asthma Therapy. British Journal of Pharmacology 2009, 157, 1330-1339.

(245) McNulty, M. M.; Edgerton, G. B.; Shah, R. D.; Hanck, D. A.; Fozzard, H. A.; Lipkind, G. M. Charge at the Lidocaine Binding Site Residue Phe-1759 Affects Permeation in Human Cardiac Voltage-Gated Sodium Channels. Journal of Physiology-London 2007, 581, 741-755.

(246) Bhattacharji, A.; Klett, N.; Go, R. C. V.; Covarrubias, M. Inhalational Anaesthetics and NAlcohols Share a Site of Action in the Neuronal Shaw2 K-V Channel. British Journal of Pharmacology 2010, 159, 1475-1485.

(247) Bhattacharji, A.; Kaplan, B.; Harris, T.; Qu, X. G.; Germann, M. W.; Covarrubias, M. The Concerted Contribution of the S4-S5 Linker and the S6 Segment to the Modulation of a K-V Channel by 1-Alkanols. Molecular Pharmacology 2006, 70, 1542-1554.

(248) Lu, Z.; Klem, A. M.; Ramu, Y. Coupling between Voltage Sensors and Activation Gate in Voltage-Gated K+ Channels. Journal of General Physiology 2002, 120, 663-676.

(249) Barber, A. F.; Liang, Q. S.; Amaral, C.; Treptow, W.; Covarrubias, M. Molecular Mapping of General Anesthetic Sites in a Voltage-Gated Ion Channel. Biophysical Journal 2011, 101, 1613-1622.

(250) Liang, Q. S.; Anderson, W. D.; Jones, S. T.; Souza, C. S.; Hosoume, J. M.; Treptow, W.; Covarrubias, M. Positive Allosteric Modulation of Kv Channels by Sevoflurane: Insights into the Structural Basis of Inhaled Anesthetic Action. Plos One 2015, 10, 20.

(251) Delemotte, L.; Tarek, M.; Klein, M. L.; Amaral, C.; Treptow, W. Intermediate States of the Kv1.2 Voltage Sensor from Atomistic Molecular Dynamics Simulations. Proceedings of the National Academy of Sciences of the United States of America 2011, 108, 6109-6114.

(252) Treptow, W.; Tarek, M. Environment of the Gating Charges in the Kv1.2 Shaker Potassium Channel. Biophysical Journal 2006, 90, L64-L66.

(253) Stock, L.; Hosoume, J.; Treptow, W. Concentration-Dependent Binding of Small Ligands to Multiple Saturable Sites in Membrane Proteins. Scientific Reports 2017, 7, 12.

(254) Jorgensen, C.; Domene, C. Location and Character of Volatile General Anesthetics Binding Sites in the Transmembrane Domain of Trpv1. Molecular Pharmaceutics 2018, 15, 39203930. 


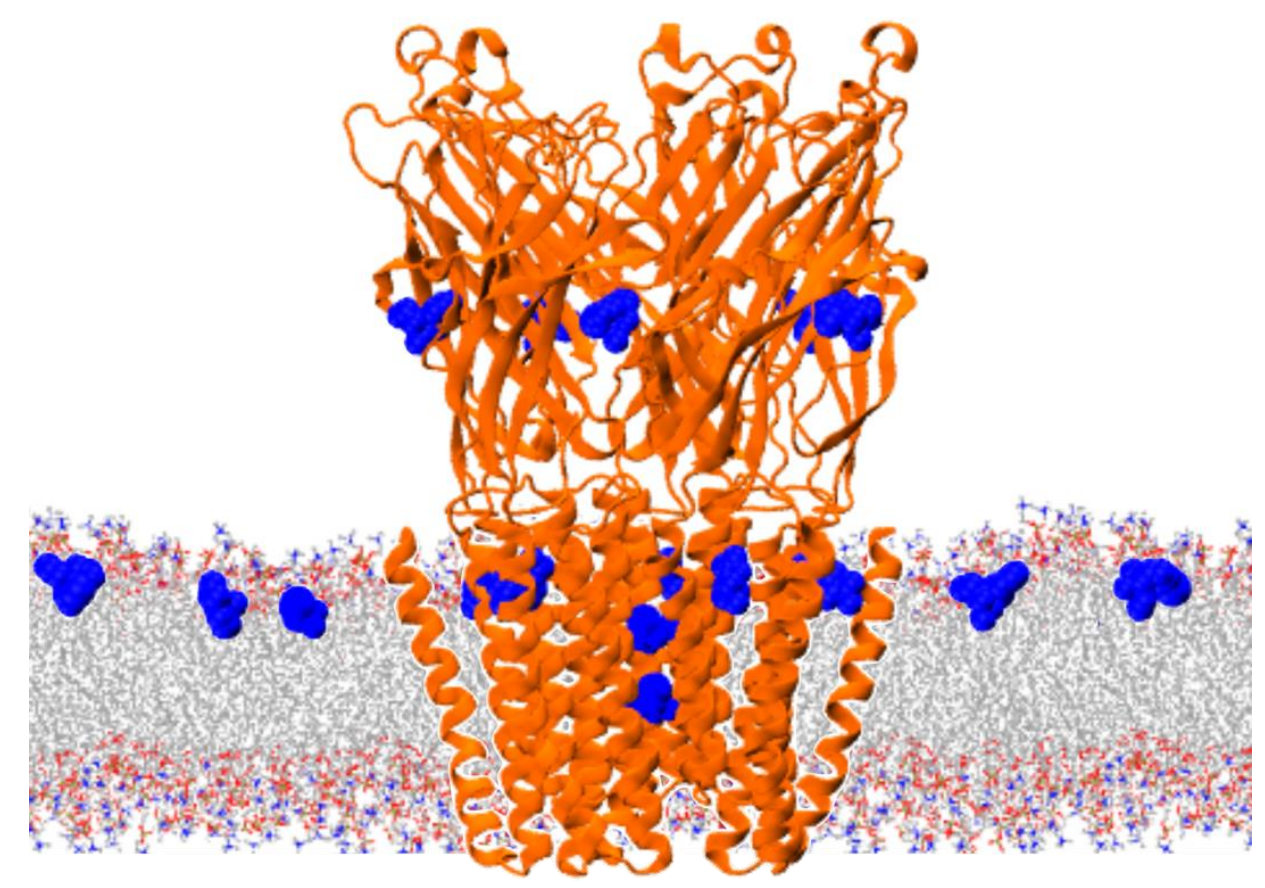

